Renate Pasch

\title{
Das „Handbuch der deutschen Konnektoren“
}

Der vorliegende Beitrag beschäftigt sich mit dem am Institut für Deutsche Sprache Mannheim (IDS) erarbeiteten „Handbuch der deutschen Konnektoren“" (= Pasch et al. 2003; im Folgenden HdK) Es beschreibt seinen Sinn und Zweck, seine Vorgeschichte und seinen Beitrag zur Beschreibung der Konnektoren im modernen Standard-Deutsch.

\section{Wozu ein ,Handbuch der deutschen Konnektoren“}

Konnektoren (wie z.B. aber, dennoch, obwohl, denn) - die in der Literatur unter den Termini „Konjunktionen“, (relationale) „Adverbien“ und „Partikeln“ behandelt werden - sind wichtige Bausteine für die Verdeutlichung der inhaltlichen Strukturierung von Texten. Ihre operationalisierbare Beschreibung ist wegen der unanschaulichen Natur der Konnektorenbedeutungen besonders für den Zweitsprachenerwerb, aber auch für die Rhetorik gefordert. Deshalb ist die Vermittlung der Gebrauchsbedingungen der Konnektoren durch Wörterbücher und Grammatiken für den muttersprachlichen, vor allem aber für den fremdsprachlichen Deutschunterricht von entscheidender Bedeutung. Trotzdem bilden Konnektoren einen Wortschatzbereich, dem in der Linguistik lange Zeit weniger Aufmerksamkeit geschenkt wurde als anderen Wortklassen (vgl. schon Lang 1982 und 1989). Insbesondere wurde seine Strukturierung in syntaktische Klassen von Grammatikographie und Lexikographie bisher recht stiefmütterlich bis irreführend behandelt. (Letzteres besonders in Wörterbüchern; s. hierzu Brauße 1997 und 2002 sowie Pasch 1994a und Kempcke/ Pasch 1998). Außerdem werden in Wörterbüchern auch die inhaltlichen Bedingungen für den korrekten Gebrauch der Konnektoren oft so beschrieben, dass ihre Beachtung bei der Verwendung des betreffenden Konnektors ohne Zuhilfenahme weiterer, nicht dokumentierter Informationen noch keine korrekte Verwendung garantiert. (Eine entsprechende Kritik findet sich bereits bei Lang 1982.) 


\section{Vorgeschichte des „Handbuchs der deutschen Kon- nektoren"}

Den Unzulänglichkeiten insbesondere der Beschreibung der inhaltlichen Seite der Konnektoren abzuhelfen war das Ziel von Arbeiten zu einzelnen Konjunktionen, semantischen Klassen von Konjunktionen, relationalen Adverbien und Partikeln oder Gruppen von diesen, die seit den siebziger Jahren des 20. Jahrhunderts verstärkt vorgelegt wurden. Die betreffenden Titel können hier aus Platzgründen nicht im Einzelnen genannt werden. Sie sind der bibliographischen Datenbank zum Thema Konnektoren zu entnehmen, die die Projektgruppe „Handbuch der deutschen Konnektoren“ erarbeitet hat und die unter der Adresse

http://www.ids-mannheim.de/gra/konnektoren/anfrage.html

zur Verfügung steht. Erwähnt seien hier nur die Bibliographie zu Partikeln von Weydt/Ehlers (1987), die Sammelbände von Fritsche (1982), Weydt (1969), (1977), (1979), (1981), (1989) sowie das „Abtönungswörterbuch“ von Weydt/ Hentschel (1983) und die „Partikellehre“ von Weydt et al. (1989), von denen sich die meisten allerdings (wie teilweise schon der Name sagt) nur auf den Teilbereich der Konnektoren bezogen, die traditionell syntaktisch als „Partikeln" klassifiziert werden. Die Einzeluntersuchungen befassten sich mit unterschiedlichen Aspekten der Konnektoren: textstrukturell-pragmatischen, lexikologisch-semantischen, lexikografischen und didaktischen. Von den traditionell angenommenen semantischen Konnektorenklassen wurden besonders kausale, adversative, konzessive und konditionale Konnektoren in ihren gängigsten Vertretern untersucht.

Die betreffenden Arbeiten behandelten allerdings nicht alle Phänomene, die für die Beschreibungen von Konnektoren relevant sind. Eine umfassende Darstellung der systematischen Züge in den Gebrauchsbedingungen aller Konnektoren des Deutschen schien aber aus dem Bedürfnis der Verbesserung der grammatiko- und lexikographischen Beschreibung der Konnektoren dringend erforderlich. Dieses Bedürfnis wurde auch in einem von Ewald Lang geleiteten „Funktionswörterzirkel“ artikuliert, der am Zentralinstitut für Sprachwissenschaft der Akademie der Wissenschaften der DDR in den achtziger Jahren des 20. Jahrhunderts Grammatiker, Lexikologen, Lexikographen und Logiker zu Diskussionen über die Beschreibung derjenigen Einheiten zusammenführte, die wir „Konnektoren“ nennen.

Nach der Abwicklung der Akademie der Wissenschaften der DDR und ihrer Institute fanden im Jahre 2002 zwei der Teilnehmer des Funktionswörterzirkels - Ursula Brauße und Renate Pasch - eine neue Arbeitsmöglichkeit am IDS Mannheim. Institutsleitung - Gerhard Stickel und Rainer Wimmer - sowie die Leiterin der Abteilung Grammatik - Gisela Zifonun - gaben, nachdem U. Brauße und R. Pasch eine Konzeption für ein Handbuch der deutschen Konnektoren vorgelegt hatten, der Bitte um Integration eines solchen Projekts 
in die Abteilung Grammatik statt. Die Abteilung Grammatik war zu diesem Zeitpunkt im Begriff, ihre in den vergangenen 10 Jahren erarbeitete Grammatik (Grammatik der deutschen Sprache in 3 Bänden = GDS $-\mathrm{s}$. Zifonun/Hoffmann/Strecker et al. 1997) zu publizieren. Das geplante Konnektorenhandbuch wurde als ein sog. Satellitenprojekt zur fertiggestellten GDS zwecks Spezifizierung der Darstellung spezieller Wortschatzbereiche konzipiert und in den Arbeitsplan des IDS aufgenommen. Nach einer Phase der Orientierung an dieser Grammatik, Einzeluntersuchungen zu semantischen Teilbereichen der Konnektoren (s. Brauße 1994d - mit den Beiträgen Brauße 1994a bis c - und Pasch 1994b) sowie einer Skizze des syntaktischen Beschreibungsinventars begannen 1993 die Arbeiten am Handbuch (dessen Inangriffnahme auch nach Erscheinen des ersten Bandes des deutsch-französischen Funktionswörterbuchs von Métrich/Faucher/Courdier - 1993; 1995; 1998 und 2002 - nicht überflüssig geworden war). 1994 lagen die endgültige Feinkonzeption des Handbuchs und erste Manuskripte zu einzelnen Handbuchabschnitten vor. 1996 stieß Eva Breindl zu den Handbuchautorinnen und übernahm u.a. die Integration der erarbeiteten Untersuchungsergebnisse in das von Bruno Strecker am IDS Mannheim entwickelte Hypertextprojekt zur GDS - „GRAMMIS“. Bereits mit Beginn des Projekts wurde Ewald Lang als kritischer Berater gewonnen. Einzelne im Handbuch zu behandelnde Teilthemen wurden in ersten Fassungen auch mit Kollegen der Abteilung Grammatik und insbesondere mit deren Leiterin - Gisela Zifonun - diskutiert sowie mit dem Mitglied des IDS-Kuratoriums Catherine Fabricius-Hansen. Da sich die Probleme der Beschreibung der Gebrauchsbedingungen, insbesondere der formalen, wegen fehlender Vorarbeiten als weitaus schwieriger und in ihrer Lösung aufwendiger erwiesen als bei der ursprünglichen Planung des Projekts angenommen, wurde der anfänglich auf fünf Jahre angesetzte Bearbeitungszeitraum verlängert und die Projektgruppe ab dem 1.4.2000 durch Ulrich Hermann Waßner verstärkt. Nach der Fertigstellung des allgemeinen semantischen und des Syntaxteils und nach der Verabschiedung von Ursula Brauße in den Ruhestand im August 2001 stieß am 1.4.2001 Hardarik Blühdorn zur Projektgruppe. Wegen der langen Bearbeitungsdauer und des Bedarfs an Übersichtsdarstellungen für den Bereich der nicht flektierbaren Lexikoneinheiten wurde beschlossen, den ersten bis dahin erarbeiteten Teil des Handbuchs unter dem oben genannten Titel „Handbuch der deutschen Konnektoren. Linguistische Grundlagen der Beschreibung und syntaktische Merkmale der deutschen Satzverknüpfer (Konjunktionen, Satzadverbien und Partikeln) " zu veröffentlichen. Hierfür stieß das IDS auf das Interesse des Verlags Walter de Gruyter, der den Band in seine Handbuchreihe aufnahm. Die Erarbeitung des allgemeinen semantischen und des Syntaxteils wurde durch Publikationen der Mitarbeiter zu einzelnen Aspekten der Konnektorenproblematik flankiert (s. hierzu neben den oben schon erwähnten Arbeiten von Brauße und Pasch die von Blühdorn 2002a, 2002b; 2003a und 2003b; Blühdorn/Meireles (Ms.); Brauße 1998; 2000; 2001; Breindl 1998; 2003a; 2003b sowie im Druck a, b und c; Pasch 1995; 1997; 1999; 2000a; 2000b und 2001 sowie Waßner 2001a, 2001b und 2002). 


\section{Zielgruppe und theoretische Grundlagen des Hand- buchs}

Das Handbuch richtet sich vornehmlich an diejenigen, die linguistische Forschungsergebnisse in Grammatiken, Wörterbüchern, Sprachtechnologie und Unterricht von Deutsch als Fremdsprache umzusetzen haben. Es soll aber auch Typologen und andere sprachtheoretisch arbeitende Linguisten interessieren. Wegen dieser Heterogenität der Zielgruppe wurde versucht, alle für die $\mathrm{Be}$ schreibungen der Gebrauchsbedingungen von Konnektoren einschlägigen Phänomene zu illustrieren und die für die Beschreibung notwendigen Begriffe in einer nicht formalen Sprache zu definieren. Als Basis für die Beschreibung der Formseite der Konnektoren wurde - ähnlich wie bei der GDS - eine Grammatik mit Phrasentypen (in der GDS: „Konstruktionskategorien“) als hierarchisch-syntaktische Kombinatorik mit Regeln der Zuordnung syntaktischer Funktionen zu Phrasentypen (die in der GDS vereinfacht gesagt „Kombinationskategorien“ genannt werden) sowie mit separaten Reihenfolgeregeln zugrunde gelegt. Die Konnektorensemantik wurde - ebenfalls informal - auf der Unterscheidung von Funktoren und ihren Argumenten, der Strukturierung von Propositionen (als Satzbedeutungen) in Hintergrund bzw. Präsuppositionen vs. Fokus sowie auf der Unterscheidung von Propositionen und einstellungsbewerteten Propositionen sowie Illokutionen konzipiert. Die einschlägigen Begriffsbildungen wurden ausführlich erläutert, um bei der Beschreibung der Konnektoren die Beschaffung umfangreicher Grundlagenliteratur überflüssig zu machen.

\section{Methodik}

Für die Ermittlung der Gebrauchsbedingungen der Konnektoren wurden neben der eigenen Sprachkompetenz (gegebenenfalls gestützt durch Kollegenbefragung) auch Belege der Konnektorenverwendung kompetenter deutscher Sprecher herangezogen. Die meisten Belege stammen aus den am IDS Mannheim zusammengestellten elektronischen Korpora. Zur Illustration der Aussagen über die Gebrauchsmöglichkeiten der Konnektoren im Handbuch wurden jedoch neben solchen Belegen aus Gründen der Systematik und der Vermeidung nicht vertretbaren Aufwands bei der Belegsuche auch selbst gebildete Beispiele verwendet. 


\section{Struktur des Handbuchs}

Eingangs werden unter den Merkmalen M1 bis M5 tentativ und prätheoretisch die Kriterien bestimmt, nach denen ein Ausdruck $x$ ein Konnektor im Sinne des Handbuchs ist:

(M1) $x$ ist nicht flektierbar.

(M2) $x$ vergibt keine Kasusmerkmale an seine syntaktische Umgebung.

(M3) Die Bedeutung von $x$ ist eine zweistellige Relation.

(M4) Die Relate der Bedeutung von $x$ sind Sachverhalte.

(M5) Die Relate der Bedeutung von $x$ müssen durch Sätze bezeichnet werden können.

M1 grenzt Konnektoren von Verben ab, M2 von Verben und Präpositionen, M3 von einstelligen Satzadverbien (wie notwendigerweise; vielleicht, tatsächlich). M4 grenzt Verwendungen bestimmter Ausdrücke als Konnektor (s. dagegen in (1)) von nichtkonnektoralen Verwendungen ab (s. dagegen in (2), wo $d a$ - einen Gegenstand bezeichnet und gegen eine lokale Beziehung zwischen zwei Gegenständen - eine Leiter und einen Baum):

(1) Gestern war es noch ziemlich kalt. Dagegen ist es heute direkt frühlingshaft.

(2) Der Baum ist zu schwach. Du solltest die Leiter nicht dagegen lehnen.

M5 grenzt Konnektoren von Infinitive regierenden Ausdrücken wie $u m z u \mathrm{ab}$, die bekanntermaßen keine Sätze regieren können. Vgl. (3):

Hans hält an um zu tanken. (*Hans hält an um zu er tankt.)

Die Formulierung „müssen durch Sätze bezeichnet werden können“ in M5 wurde aufgrund folgender Phänomene gewählt:

(i) Als Bezeichnungen der Relate der Konnektorenbedeutungen - d.h. als „Konnekte“ - kommen nicht nur Sätze in Frage, sondern auch Einheiten, die keine Sätze sind, aber in der jeweiligen Verwendung des Konnektors auch jeweils zu einem Satz erweitert werden können. Dies betrifft Fälle der Verwendung koordinierender (vgl. und) oder in eines ihrer Konnekte integrierter (vgl. folglich) Konnektoren, aber auch Fälle der Verwendung mancher subordinierenden Konnektoren (wie weil). Vgl. die Konstruktionen unter (4):

(4a) Dort wächst noch Frühlingsenzian und Almenrausch.

(4b) Das ist zu langwierig, folglich ist das nicht machbar. 
Die Rosen haben nur drei Fage in der Vase gehalten, aber die Nelken haben vier Tage in der Vase gehalten. Die Rosen haben nur drei Tage in der Vase gehalten, aber die Nelken haben vier Tage in der Vase gehatten.

Weil ex seit langem krank sagte er alle Termine ab.

Die Konstruktionen unter (4) sind als Ergebnisse von (hier mittels Durchstreichungen symbolisierten) Weglassungen lautlicher Realisierungen von Konstituenten abstrakter „Satzstrukturen“ zu interpretieren.

(ii) Konnektoren können auch attributiv verwendete Adjektiv- bzw. Partizipialphrasen, also „Nicht-Satzstrukturen“ verknüpfen:

(5a) der, weil an Grippe erkrankte, nicht von der Sitzung informierte Kollege

(5b) die, obwohl unbeliebte, schon zum zweiten Mal wiedergewählte Kandidatin

(5c) der weder schöne noch charmante Schauspieler

(5d) die von allen verehrte, jedoch Eiemlich kühle Sängerin

Um den unter (4) und (5) illustrierten Konnektorenverwendungen Rechnung zu tragen, wurde M5 später modifiziert zu M5':

(M5') Die Ausdrücke für die Argumente der Bedeutung von $x$ müssen Satzstrukturen sein können.

Dabei wird die relationale Bedeutung eines Konnektors als Funktor betrachtet und die Relate als dessen Argumente.

Die Formulierung von M4 erwies sich bei tiefer gehenden Untersuchungen ebenfalls als nicht treffend genug angesichts des folgenden Phänomens: Die Relate der Konnektorenbedeutung müssen nicht unbedingt Sachverhalte sein. Sie können neben Sachverhalten - auf der Ebene der Beschreibung der Konnektbedeutungen sind dies Propositionen (als Sachverhalte identifizierende Bedeutungseinheiten) (vgl. (6a)) - auch ,epistemische Minimaleinheiten“ (vgl. (6b)) oder Illokutionen (sprachliche Handlungen) (vgl. (6c)) sein:

(6a) Weil es Frost gegeben hat, sind die Dahlien ganz schwarz. (Die Argumente sind Propositionen: Frost wird als Ursache für die Verfärbung der Dahlien hingestellt.)

(6b) Es hat Frost gegeben, denn die Dahlien sind ganz schwarz. (Die Argumente sind epistemische Minimaleinheiten: Die Annahme, dass es Frost gegeben hat, wird mit dem Inhalt der Behauptung begründet, dass die Dahlien schwarz sind.) 
(6c) Du, morgen ist Ratssitzung, da musst du nämlich hingehen. (Das erste Argument ist eine Illokution: Die Mitteilung, dass am Folgetag Ratssitzung ist, wird damit begründet, dass der Adressat der Äußerung zu dieser Sitzung gehen muss. Es wird unterstellt, dass er von der Sitzung keine Kenntnis hatte.)

Im Handbuch werden die inhaltlichen Einheiten „Proposition“, „epistemische Minimaleinheit“ und „Illokution“ unter dem Ausdruck „propositionale Strukturen" zusammengefasst und aufgrund der in (6) illustrierten unterschiedlichen Typen von Argumenten der Bedeutung bestimmter Konnektoren wurde die Formulierung von M4 entsprechend zu M4' verfeinert:

(M4') Die Argumente der Bedeutung von $x$ sind propositionale Strukturen.

Im Anschluss an die Eingrenzung des Handbuchgegenstands werden die Phänomene behandelt, die für die Beschreibung der Gebrauchsbedingungen der Konnektoren einschlägig sind. Diese Phänomene sind in der nachfolgenden Übersicht über die Struktur des Handbuchs mit seinen Hauptthemenfeldern angeführt.

\section{A Bestimmung des Handbuchgegenstands}

Kriterien für die Abgrenzung des Gegenstands, einführende Bemerkungen zum Zusammenspiel inhaltlicher und syntaktischer Gebrauchsbedingungen von Konnektoren und zum Verhältnis von semantischer und syntaktischer Klassifikation der Konnektoren

\section{B Linguistische Grundlagen für die Beschreibung der Konnektoren, Begriffsbildung und Definitionen}

1. Ausführungen zum Verhältnis von Sprachsystem und Sprachverwendung (Ausdrücke vs. Ausdrucksakte; grammatisch determinierte Bedeutung vs. Äußerungsbedeutung; zum Begriff der „Gebrauchsbedingungen“; zu Typen von Referenz gemäß syntaktischen Kategorien; zur Unterscheidung von „wörtlicher“" und ,nichtwörtlicher“ Äußerungsbedeutung)

2. Einführung in die Prinzipien syntaktischer Strukturbildung (syntaktische Kategorisierung sprachlicher Ausdrücke: Konstituentenkategorien und Konstituentenstruktur; Konnektoren im System der lexikalischen Kategorien (Wortarten); phrasale Konstituentenkategorien und ihre Binnenstruktur; Konnektoren und Phrasen; syntaktische Funktionen (nicht-finite Begleiter des Verbs - Komplemente und Supplemente -, Attribute); Konnektoren und syntaktische Funktionen; zum Zusammenhang syntaktischer Konstituentenkategorien und syntaktischer Funktionen; linear-syntaktische Strukturierung sprachlicher Ausdrücke: Satzklammer und Felder; Linearstruktur vs. Satzstruktur: Herausstellungen und appositive Einschübe; intonatorische Ausformung von 
Ausdrücken: Akzentuierung, „Intonation”; Bestimmung der Begriffe „Satzstruktur" und „Satz"; topologische Satztypen)

3. Einführung in die Prinzipien der Strukturierung von Interpretationen (Funktor-Argument-Struktur; Termbedeutungen vs. Prädikate; FokusHintergrund-Gliederung (Fokus-Hintergrund-Gliederung komplexer Sätze, fokus-sensitive Ausdrücke); präsuppositionale Propositionen vs. Hauptproposition (logische vs. nichtlogische Präsuppositionen, grammatisch vs. textuell induzierte Präsuppositionen, Präsuppositionen als Argumente von Konnektoren); epistemischer Modus und propositionaler Gehalt (epistemischer Modus von Satzstrukturen und von Nominalphrasenbedeutungen, epistemischer Modus von Präsuppositionen); epistemische Minimaleinheiten und Konnektoren; Äußerungsbedeutung und kommunikative Funktion von Äußerungen; „Satzillokution“ vs. „sekundäre Illokutionen“; konzeptuelle Strukturen: Nivellierung ausdrucksbedingter Unterschiede in Aspekten der Ausdrucksinterpretation)

4. Epistemische Modi und Satzmodi (epistemische Modi, Satzmodi und Konnektorenbeschränkungen; Satzmodi - epistemische Modi - kommunikative Funktionen)

5. Syntax der komplexen Satzstrukturen und „Hauptsatzphänomene“ (Subordination und Einbettung; zum Verhältnis von Einbettung zu Einschub und Nachtrag; Korrelatkonstruktionen (attributive Korrelatkonstruktionen vs. Versetzungskonstruktionen): zur Frage der syntaktischen Beziehung zwischen Korrelat und versetztem Ausdruck; Korrelate und Konnektoren; syntaktische Desintegration, Koordination (kontinuierliche und diskontinuierliche koordinative Verknüpfungen; topologische Unterschiede zwischen Satzstrukturkoordinaten; zur Rolle der Reihenfolge der Koordinate, zu den Akzentverhältnissen in koordinativen Konstruktionen; Konnexion und Kollektion; Koordinationsbeschränkungen); zum Verhältnis zwischen „komplexen Satzstrukturen“ und „Parataxe“)

6. Das Phänomen der Ellipse (u.a. Weglassungsbeschränkungen)

7. Merkmale von Konnektoren (verfeinerte Konnektorenkriterien)

8. Phraseologische Konnektoren

9. Zur Behandlung frei bildbarer, d.h. ableitbarer syntaktisch komplexer Konnektoren (konnektintegrierbare Konnektoren: angesichts dessen; anhand dessen; anstellelan Stelle dessen; aufgrund/auf Grund dessen; im/in Hinblick darauf, in Anbetracht dessen; in/mit Bezug darauf, infolgedessen; in Übereinstimmung damit; unbeschadet dessen; ungeachtet dessen; vorbehaltlich dessen; Subjunktoren: angesichts dessen, dass; anhand dessen, dass; anstellelan Stelle dessen, dass; aufgrund/auf Grund dessen, dass; für den Fall (...), dass; im Fall(e) (...), dass; im/in Hinblick darauf, dass; in Anbetracht dessen, dass; in/mit Bezug darauf, dass; infolgedessen, dass; in Übereinstimmung damit, dass; mit dem Ziel (...), dass; unbeschadet dessen, dass; ungeachtet dessen, dass; unter der Bedingung (...), dass; unter der Voraussetzung (...), dass und vorbehaltlich dessen, dass; Unterscheidung zweier Typen von Ableitungen frei bildbarer Subjunktoren: attributive Erweiterungen eines Pronominaladverbs oder Pronomens - z.B. bei im Hinblick darauf, dass oder anhand dessen, dass 
- vs. attributive Erweiterungen von Nomina in Präpositionalphrasen, die als phraseologische Präpositionen fungieren - z.B. für den Fall (...), dass)

\section{Syntaktische Konnektorenklassen}

\section{Nichtkonnektintegrierbare (konjunktionale) Konnektoren}

1.1 Subjunktoren (Liste der nicht frei bildbaren Subjunktoren und Verwendungsbeispiele; Nichtsatzsubjunkte als Ergebnisse von Weglassungen; attributiv verwendete Adjektiv- und Partizipialkonnekte von Subjunktoren; Hauptsätze als internes Konnekt; die Beziehung zwischen Einbettung einerseits und Fokus-Hintergrund-Gliederung sowie prosodischer Form der Subjunktorkonstruktionen andererseits; die Position der Subjunktorphrase in der linearen Ordnung der Subjunktorkonstruktion; syntaktische Funktionen von Subjunktorphrasen, u.a. Subjunktorphrasen als Komplemente (Ergänzungen von Prädikatsausdrücken); Subjunktorphrasen mit Versetzungskorrelat; syntaktisch desintegrierte Subjunktorphrasen; prosodisch manifeste Desintegration der Subjunktorphrase; semantische Desintegration von Subjunktorphrasen; syntaktisch selbständig verwendete Subjunktorphrasen; Subjunktoren und ihre Korrelate; Subjunktoren als parataktische Konnektoren; syntaktische Merkmale der Subjunktoren)

1.2 Postponierer (Liste der Postponierer und Verwendungsbeispiele; syntaktische Merkmale der Postponierer)

1.3 Verbzweitsatz-Einbetter (Liste der Verbzweitsatz-Einbetter und Verwendungsbeispiele; topologische Phänomene; Fokus-Hintergrund-Gliederung von Konstruktionen mit Verbzweitsatz-Einbettern; zur Herkunft der Verbzweitsatz-Einbetter aus anderen syntaktischen Kategorien; syntaktische Merkmale der Verbzweitsatz-Einbetter)

1.4 Konjunktoren (Liste der Konjunktoren und Verwendungsbeispiele; zur Frage der Weglassbarkeit von Konjunktoren; zum Verbstellungstyp der Konnekte; zur Stellung der Teile mehrteiliger Konjunktoren; syntaktische Merkmale der Konjunktoren)

2. Konnektintegrierbare (adverbiale) Konnektoren (Prinzipien der Klassenbildung bei konnektintegrierbaren Konnektoren; Fragen des Gegenstandsbereichs und der Terminologie; Syntax der konnektintegrierbaren Konnektoren (zur Funktion der konnektintegrierbaren Konnektoren als Satzmodifikatoren); mehrteilige konnektintegrierbare Konnektoren; syntaktische Eigenschaften der Konnekte konnektintegrierbarer Konnektoren; konnektintegrierbare Konnektoren als Verknüpfer elliptischer Konnekte; syntaktische Einzelgänger im Bereich der konnektintegrierbaren Konnektoren; Übersicht über die nicht frei bildbaren konnektintegrierbaren Konnektoren mit Angabe ihrer syntaktischen Klasse und Positionsmöglichkeiten)

\subsection{Nicht positionsbeschränkte Adverbkonnektoren}

2.2 Nicht nacherstfähige Adverbkonnektoren (Pronominaladverbien in Konnektorfunktion; Wortbildung bei Pronominaladverbien; akzentuelle Varianz bei Pronominaladverbien) 
2.3 Nicht vorfeldfähige Adverbkonnektoren (Fokuspartikeln, „Abtönungspartikeln")

3. Einzelgänger (Begründungs-denn; es sei denn; außer; geschweige (denn); kaum als temporaler Konnektor; als mit folgendem konjunktivischem Verberstsatz; sei es; $o b$ in desintegrierten Alternativenausdrücken; begründendkausales dass; ausgenommen; je nachdem; statt und anstatt)

4. Matrix der syntaktischen Konnektorenklassen und der klassenbildenden Merkmale

\section{Syntaktische Polykategorialität von Konnektoren}

D Konnektorenliste (Liste aller im Text erwähnten Konnektoren - d.h. sowohl der nicht frei bildbaren als auch der frei bildbaren - mit Angabe der syntaktischen Klasse, der sie angehören; hier wird zu jedem Konnektor ein Verwendungsbeispiel angeführt und bei manchen Konnektoren auch ein stilistisches Merkmal; die syntaktischen Merkmale, die sich hinter dem Namen der syntaktischen Klasse verbergen, sind über das Sachregister zu ermitteln; ihr zentraler Platz im Handbuchtextteil ist im Sachregister durch Fettdruck gekennzeichnet)

\section{Beitrag des Handbuchs zur Konnektorenforschung}

\subsection{Defizite in der Behandlung der Konnektorensyntax}

Ein wesentlicher Beitrag des syntaktischen Teils des Handbuchs ist in seinem Vorschlag zu einer Neuordnung der syntaktischen Klassifikation der Konnektoren zu sehen. Dieser macht das Herzstück des vorliegenden Handbuchbandes aus. Der Neuordnungsvorschlag versucht, das Fehlen scharfer operationalisierbarer Kriterien für die syntaktische Klassifikation von Konjunktionen sowie adverbialen Konnektoren und Partikeln zu beheben, das die bisherige Literatur zu Konnektoren kennzeichnet. Bei der Arbeit am Konnektorenhandbuch zeigte sich nämlich immer deutlicher, dass die Kriterien für die tradierten Ergebnisse syntaktischer Klassifizierung sowohl a) im Bereich der traditionell als „subordinierend" bezeichneten Konjunktionen als auch b) im Bereich der satzintern zu verwendenden Konnektoren - „Adverbien“ und „Partikeln“ - als auch c) zwischen Letzteren und den traditionell als „koordinierend“ analysierten Konjunktionen mehr oder weniger unscharf sind und damit die Angabe einer syntaktischen Klasse bei einem Ausdruck nicht genau genug umreißt, wie der Konnektor korrekt zu verwenden ist.

So ist z.B. nicht klar, wie in der traditionellen Behandlung der Konnektoren die Kriterien für „subordinierende“ (,hypotaktische“) Konjunktionen aussehen: Es werden z.B. (wie noch in der GDS) Verbzweitsätze als eines ihrer Konnekte regierende Konnektoren (wie angenommen oder vorausgesetzt; vgl. (8)) mit Verbletztsätze regierenden Konjunktionen (wie als; bevor; da oder wenn; vgl. (7)) in einer Klasse zusammengefasst. 
(7a) Wenn es nicht regnet, machen wir einen Ausflug.

(7b) Wir machen, wenn es nicht regnet, einen Ausflug.

(7c) Wir machen einen Ausflug, wenn es nicht regnet.

(8a) Vorausgesetzt es regnet nicht, machen wir einen Ausflug.

(8b) Wir machen, vorausgesetzt es regnet nicht, einen Ausflug.

(8c) Wir machen einen Ausflug, vorausgesetzt es regnet nicht.

Die Unterschiede zwischen den Konstruktionen unter (7) und denen unter (8) in Bezug auf die Position des Verbs im regierten Satz als dem ,internen Konnekt" des Konnektors sind wichtige Gebrauchsbedingungen der betreffenden Konnektoren.

Des Weiteren werden traditionell Konjunktionen, die einen Verbletztsatz als ihr internes Konnekt regieren, aber mit diesem nicht das Vorfeld ihres anderen - „externen“ - Konnekts besetzen können (wie sodass; vgl. (9a) vs. (9b) und (9c)), mit Konjunktionen, die mitsamt dem von ihnen regierten Verbletztsatz als ihrem internen Konnekt das Vorfeld ihres externen Konnekts besetzen können (wie als; bevor; da oder wenn; vgl. (6)), unter ein und dieselbe Klasse „subordinierende“ Konjunktionen subsumiert (wie z.B. in der GDS).

(9a) Jemand schoss in die Luft, sodass alle sich auf den Boden warfen.

(9b) *Sodass alle sich auf den Boden warfen, schoss jemand in die Luft.

(9c) ?Jemand schoss, sodass alle sich auf den Boden warfen, in die Luft.

Ferner werden traditionell nicht Konjunktionen wie und oder oder, die einbettbare Satzverknüpfungen bilden können (vgl. (10)), von zwischen seinen Konnekten auftretendem denn unterschieden (vgl. (11)). Diese werden ohne Unterschied als „koordinierend“ beschrieben. Eine derartige Angabe im Wörterbuch wird jedoch, wie (11) im Kontrast zu (10) zeigt, dem unterschiedlichen Verhalten der betreffenden Konnektoren bei Einbettung der Konnektorkonstruktion nicht gerecht.

(10a) Weil es immer anders kommt, als man denkt, und niemand dem Schicksal vorgreifen kann, bin ich ziemlich gelassen.

(10b) Bist du traurig, weil es immer anders kommt, als man denkt und niemand dem Schicksal vorgreifen kann?

(11a) *Weil es immer anders kommt, als man denkt, denn niemand das Schicksal beeinflussen kann, bin ich ziemlich gelassen.

(11b) *Bist du traurig, weil es immer anders kommt, als man denkt, denn niemand kann das Schicksal beeinflussen?

Des Weiteren ist nicht klar, nach welchen Merkmalen die relationalen Satzadverbien und Partikeln syntaktisch zu klassifizieren sind. Begriffsbestimmungen wie „dient der Hervorhebung“, „Würzwort“ oder „Abtönungspartikel“ sind keine syntaktischen Kriterien, weil sie nicht konsistent auf topologische und/ oder syntaktisch-hierarchische Phänomene gegründet sind. Aber auch als se- 
mantische Kriterien sind sie viel zu vage, um von Wörterbuchnutzern produktiv gemacht werden zu können. Grammatiken vernachlässigen im Allgemeinen die syntaktische Klassifikation (s. aber Clément/Thümmel 1975), bieten allenfalls eine semantische an. Wörterbücher verfahren unsystematisch (s. Brauße 1997, 2002 und Kempcke/Pasch 1998).

Ein weiteres Defizit der bisherigen Behandlung von Konnektoren in Grammatiken und Wörterbüchern ist die Vernachlässigung von Unterschieden zwischen Klassen von Konnektoren im Hinblick auf die Fokus-HintergrundGliederung der durch die Konnektoren hergestellten Satzverknüpfungen, Unterschiede, die durchaus zu den Gebrauchsbedingungen der Konnektoren gehören. Vgl. die Beispiele für kontextuell angemessene Verwendungen von Konstruktionen mit einem Konnektor eines bestimmten syntaktischen Typs unter (12) im Unterschied zu den kontextuell nicht angemessenen Verwendungen von Konstruktionen mit einem Konnektor eines anderen syntaktischen Typs unter (13):

(12a) [A.: Morgen regnet es, sagt der Wetterbericht. B.:] Wenn \{es morgen regnet $\} \mathrm{H}$, \{gehen wir ins Museum\}F./\{Dann gehen wir ins Muse$u m\} \mathrm{F}$, wenn es morgen regnet $\} \mathrm{H}$.

(12b) [A.: Wann geht ihr denn ins Museum? B.:] \{Wir gehen ins Muse$u m\} \mathrm{H}$, wenn $\{$ es morgen regnet $\} \mathrm{F} /$ Wenn $\{$ es morgen regnet $\} \mathrm{F}$, \{gehen wir ins Museum \}H.

(13a) [A.: Du bist ja verletzt! B.:] ? \{lch bin verletzt $\} \mathrm{H}$, sodass $\{$ ich zum A rzt muss\}F.

(13b) [A.: Warum musst du denn zum Arzt? B.:] *\{Ich bin verletzt $\} \mathrm{F}$, sodass \{ich zum Arzt muss $\} \mathrm{H}$.

(Der Index „H“ steht für „Hintergrund“ und der Index „F“ für „Fokus“. Die eckigen Klammern fassen Kontext zum jeweils zu betrachtenden Ausdruck zusammen. Durch Unterstreichung kennzeichne ich den Gipfel der Silbe, die den Hauptakzent der Satzverknüpfung trägt.)

In (12a) ist die Information, die durch die unmittelbar auf wenn folgende Satzstruktur - d.h. durch das interne Konnekt des Konnektors, nämlich durch es morgen regnet, ausgedrückt wird, durch die als sprachlicher Kontext dienende Äußerung von A. vorerwähnt. Da sie mit nichts kontrastiert wird, ist sie „Hintergrundinformation“. Der Rest der Äußerung von B. - das andere Konnekt - bildet den „Fokus“ der durch wenn hergestellten Satzverknüpfung. In (12b) wird Hintergrundinformation in der Äußerung von B. durch wir gehen ins Museum bzw. gehen wir ins Museum ausgedrückt. Bei Konnektoren wie wenn können also beide Konnekte Hintergrundinformation ausdrücken. Dadurch sind sie prädestiniert zum Ausdruck von Information, die als evident für den Adressaten der Äußerung zu unterstellen ist, dabei zum Ausdruck von auch textuell induzierten - Präsuppositionen, wie z.B. der für das interne Konnekt das so ist von weil in (14) induzierten Präsupposition: 
Die Thematik ist doch viel zu komplex. Weil das so ist, muss man viel mehr Zeit für das Projekt veranschlagen als geplant.

Diese Präsupposition besagt, dass die Thematik, auf die mit der Satzstruktur das so ist Bezug genommen wird, viel zu komplex ist.

Für Konnektoren wie sodass ist, wie die Kontextunangemessenheit der Konstruktionen unter (13) zeigt, die Möglichkeit, dass eines ihrer Konnekte Hintergrundinformation ausdrückt, nicht gegeben: Bei sodass müssen beide Konnekte fokal sein. (13a) ist dadurch einfach kontextunangemessen verwendet - was durch das Fragezeichen vor der Satzverknüpfung signalisiert werden soll - (13b) ist darüber hinaus noch grammatisch abweichend, weil der Hauptakzent in Konstruktionen mit sodass auf das zweite Konnekt fallen muss.

\subsection{Durch das Handbuch eingebrachte Neuerungen in der Konnekto- renbeschreibung}

Das Handbuch leistet einen eigenständigen Beitrag zur Grammatikforschung, und zwar in zweierlei Hinsicht: Zum einen bietet es eine Revision der in der Grammatikliteratur üblichen syntaktischen Konnektorenklassifikationen an. Zum anderen trägt es durch integrative und teilweise neuartige Behandlung der sich in den Gebrauchsbedingungen von Konnektoren manifestierenden Phänomene zur Diskussion allgemeingrammatischer Fragen bei.

\subsubsection{Die syntaktische Klassifikation der Konnektoren}

Das Handbuch räumt die in 6.1 genannten Mängel der traditionellen Konnektorenklassifikationen aus. Es legt den syntaktischen Klassen, die es unterscheidet, überprüfbare Merkmale zugrunde. Für die Konnektoren, die traditionell als „Konjunktionen“ bezeichnet werden, sind dies die Art der Einflussnahme auf eines ihrer Konnekte, d.h. Phänomene der Rektion, und die Platzierbarkeit des Konnektors mit dem regierten Konnekt bezüglich seines anderen Konnekts. Bei den Konnektoren, die traditionell als „Adverbien“ und/oder „Partikeln" bezeichnet werden, sind dies die Möglichkeiten der Platzierung in einem ihrer Konnekte. (Auf die im Handbuch vorgeschlagenen Klassen und die die Klassen definierenden Merkmale gehe ich weiter unten noch genauer ein.) Zwecks eines Vergleichs der Leistungen der syntaktisch so unterschiedlichen Konnektoren hinsichtlich ihrer Bedeutungsbeiträge zu den durch sie hergestellten Satzstrukturverknüpfungen wurden die Konnekte der Konnektoren typisiert: Das Konnekt, das immer unmittelbar auf den Konnektor folgt bzw. in das der Konnektor integriert werden kann, wird im Handbuch unabhängig von der syntaktischen Klasse, der der Konnektor zugeordnet wird, ,internes Konnekt“" genannt, das andere Konnekt „externes Konnekt“. So ist es möglich, die semantische Rolle des internen Konnekts unterschiedlicher Konnektoren, 
die die gleiche semantische Relation ausdrücken, zu vergleichen. Weil ist ein kausaler Konnektor, weil sein internes Konnekt in einer Ursache-WirkungRelation die Ursache bezeichnen kann, sodass und deshalb sind konsekutive Konnektoren, weil ihr internes Konnekt in dieser Relation die Wirkung bezeichnen kann.

Das im Handbuch verfolgte Prinzip der Klassifikation ist die Zusammenfassung von für viele Konnektoren geltenden Merkmalen zu disjunkten Klassen zwecks ökonomischer Darstellung in Wörterbüchern. Diejenigen Konnektoren, bei denen zu viele Merkmale unterschiedlicher Klassen zusammentreffen und die in diesen Merkmalen nicht ihresgleichen haben, wurden nicht klassifiziert, sondern als „Einzelgänger“ in einem gesonderten Kapitel (C 3.) beschrieben. (Dies betrifft u.a. das zwischen seinen Konnekten auftretende denn.) Die Festlegung von Kriterien machte es mitunter erforderlich, für die Klassen neue Termini einzuführen. Diese sollen dazu dienen, Assoziationen mit bestehenden, aber inhaltlich anders gefassten Termini zu vermeiden. Dabei wurde Durchsichtigkeit der neuen Termini angestrebt, was bei diesen unvermeidlich zu einer gewissen Schwerfälligkeit führte.

Das Handbuch unterscheidet vom systematischen Standpunkt zwei Konnektoren-Großklassen, die aber lexikographisch oder grammatikographisch keinen praktischen Nutzen bringen, weil sie noch zu grob sind: konnektintegrierbare Konnektoren - das sind relationale Adverbien und Partikeln, im Handbuch auch „Adverbkonnektoren“ genannt - vs. nichtkonnektintegrierbare Konnektoren - das sind traditionell „Konjunktionen“ genannte Konnektoren. Erstere können direkte Konstituenten von Sätzen sein, Letztere nicht. Konnektintegrierbare Konnektoren unterliegen der Beschränkung auf Typen von Positionen in der Linearstruktur von Sätzen. Nichtkonnektintegrierbare Konnektoren sind nach Typen entsprechend den syntaktischen Eigenschaften (eines) ihrer Konnekte zu subklassifizieren. Die Striktheit beim Anlegen der Kriterien an einen Konnektor führte teilweise zu Umklassifizierungen bestimmter Konnektoren im Vergleich zu traditionellen Klassifizierungen. So wird z.B. weder (...) noch nicht mehr als Konjunktor (koordinierende Konjunktion) gesehen, sondern als konnektintegrierbarer, nur konnektintegriert zu verwendender Konnektor noch mit möglicher formaler Konsequenz (weder) im ersten Konnekt. Ähnliches gilt für aber, das zwischen seinen Konnekten, aber auch in sein zweites Konnekt integriert verwendet werden kann.

Die Elemente der im Handbuch angenommenen 7 syntaktischen Klassen werden im Folgenden in den Anhängen unter 7.1 aufgeführt. In 7.2 findet sich eine Matrix, in der die Klassen mit ihren Merkmalen zusammenfassend charakterisiert werden. 


\subsubsection{Syntaktische Klassen nichtkonnektintegrierbarer Konnektoren und ihre Merkmale}

Bei den nichtkonnektintegrierbaren Konnektoren unterscheidet das Handbuch folgende syntaktische Klassen: Subjunktoren (wie wenn; vgl. (7)), Verbzweitsatz-Einbetter (wie vorausgesetzt; vgl. (8)), Postponierer (wie sodass; vgl. (9)) und Konjunktoren (wie und; vgl. (10)).

Subjunktoren, Verbzweitsatz-Einbetter und Postponierer regieren ihr internes Konnekt, d.h. sie fordern für dieses eine bestimmte Form: Subjunktoren fordern, dass ihr internes Konnekt, wenn es wie in (7) in Satzform geäußert wird, ein Verbletztsatz ist. (Von der Möglichkeit, dass bei Platzierung des Konnektors mit seinem internen Konnekt im Anschluss an sein externes Konnekt das interne Konnekt Verbzweitsatzform hat - vgl. Ich nehme das Buch, weil - es ist so schön aufgemacht.; Ich nehme das Buch, obwohl - ich habe gar keinen Platz mehr. -, sehe ich hier einmal ab.) Vgl. (7) vs. (7'):

(7'a) *Wenn es regnet nicht, machen wir einen Ausflug.

(7'b) *Wir machen, wenn es regnet nicht, einen Ausflug.

(7'c) *Wir machen einen Ausflug, wenn es regnet nicht.

Postponierer fordern ebenfalls für ihr internes Konnekt, dass es ein Verbletztsatz ist: Vgl. (9a) vs. (9'a):

(9'a) *Jemand schoss in die Luft, sodass alle warfen sich auf den Boden.

Dabei dulden sie, anders als manche Subjunktoren (vgl. (4e)) keine Weglassung des finiten Verbs im internen Konnekt.

Verbzweitsatz-Einbetter fordern, dass ihr internes Konnekt ein Verbzweitsatz ist - vgl. (8) vs. ( $\left.8^{\prime}\right)$ :

(8'a) *Vorausgesetzt es nicht regnet, machen wir einen Ausflug.

(8'b) *Wir machen, vorausgesetzt es nicht regnet, einen Ausflug.

(8'c) *Wir machen einen Ausflug, vorausgesetzt es nicht regnet.

Sie lassen wie Postponierer in ihrem internen Konnekt keine Weglassung des finiten Verbs zu. Vgl. (15):

(15a) Vielleicht ist morgen schönes Wetter. Dann machen wir einen Ausflug. \{Wenn nicht ist/*vorausgesetzt ist nicht

(15b) Er ist, weil ü überall beliebt ist, zum Sprecher der Gruppe gewählt worden.

(15c) *Man wird, vorausgesetzt beliebt, leicht in ein Amt gewählt. 
Konjunktoren dagegen koordinieren ihre Konnekte: Das heißt im Einzelnen, dass sie keines ihrer Konnekte regieren, d.h. weder die Form noch die syntaktische Funktion eines oder gar beider Konnekte bestimmen. Die syntaktische Funktion der Konnekte wird von außerhalb der durch den Konnektor hergestellten semantischen Beziehung zwischen den Konnekten determiniert. Die einzige Forderung, die Konjunktoren an ihre Konnekte stellen, ist, dass die Konnekte, wenn die durch den Konjunktor hergestellte Konnektverknüpfung eingebettet ist, identische syntaktische Funktionen in dem Ausdruck ausüben müssen, in den die Verknüpfung eingebettet ist. Vgl.:

Ich frage mich, ob er kommen wird und wem er etwas mitbringen wird.

Sie besuchten sie wegen ihrer Tiere und weil sie mal wieder im Grïnen sein wollten.

Es ist völlig unklar, ob er sie besuchen oder sie zu sich einladen will.

In (16a) üben die beiden Konnekte von und die Funktion eines Akkusativkomplements (traditionell: „Akkusativobjekts“) zum Verb fragen aus, in (16b) üben sie die Funktion eines Supplements (traditionell: einer „Adverbialbestimmung") aus und in (16c) sind die beiden Konnekte von oder Kokonstituenten zu $o b$ (mit einer syntaktischen Funktion, für die es im Repertoire der in traditionellen Grammatiken unterschiedenen syntaktischen Funktionen keinen Namen gibt).

Subjunktoren und Verbzweitsatz-Einbetter betten ihr internes in ihr externes Konnekt ein (wie der Subjunktor wenn in (7) und der VerbzweitsatzEinbetter vorausgesetzt in (8)). Ein Kriterium für den einbettenden Charakter eines Konnektors ist, dass der Konnektor mitsamt seinem (ihm unmittelbar folgenden) internen Konnekt in seinem externen Konnekt an unterschiedlichen Stellen auftreten kann. Dabei ist das zentrale Kriterium, dass er mit seinem internen Konnekt das Vorfeld des externen Konnekts besetzen kann. Postponierer - wie sodass - können Letzteres nicht (vgl. (9b) und (9c)): Sie sind wie die Konjunktoren - nicht einbettende Konnektoren. Da die Postponierer aber - anders als die Konjunktoren - ihr internes Konnekt regieren, indem sie für dieses Verbletztstellung fordern (vgl. (9a) vs. (9'a)), sind sie im Bereich der nichtkonnektintegrierbaren Konnektoren ein Grenzfall zwischen Subjunktoren und Verbzweitsatz-Einbettern als regierenden Konnektoren einerseits und Konjunktoren als koordinierenden, d.h. nichtregierenden Konnektoren andererseits.

Die Postponierer gehen wie gesagt mit den Subjunktoren in der Frage ihrer Rektion zusammen. Sie unterscheiden sich aber von diesen nicht nur durch die Platzfestigkeit von Konnektor und internem Konnekt, sondern auch dadurch, dass keines ihrer Konnekte Hintergrundinformation liefern darf. Vgl. weiter oben die Beispiele für kontextuell angemessene Verwendungen von Sub- 
junktorkonstruktionen unter (12) im Unterschied zu den kontextuell nicht angemessenen Verwendungen von Postponiererkonstruktionen unter (13).

Die Gegenüberstellung von (12) und (13) zeigt, dass bei Subjunktoren mal das eine, mal das andere Konnekt Hintergrundinformation bezüglich des jeweils anderen ausdrücken kann. Dadurch sind Subjunktoren prädestiniert zum Ausdruck von Information, die als evident für den Adressaten der Äußerung zu unterstellen ist, dabei von - auch textuell induzierten - Präsuppositionen, wie in (14). Für Postponierer ist, wie die Kontextunangemessenheit der Konstruktionen mit sodass unter (13) zeigt, die Möglichkeit, dass eines ihrer Konnekte Hintergrundinformation bezüglich des anderen Konnekts ausdrückt, nicht gegeben: Bei Postponierern darf die Bedeutung keines der Konnekte Hintergrund für die Bedeutung des jeweils anderen sein. Das schließt nicht aus, dass Teilausdrücke der beiden Konnekte Hintergrundinformation bezüglich des Kontextes der Konnektorkonstruktion ausdrücken können. Vgl.:

[A.: Wer wird denn die Aufgabe übernehmen? B.:] \{\{Die wird $\} \mathrm{H}$ $\{$ Lucie $\} \mathrm{F}$ \{übernehmen $\} \mathrm{H}\} \mathrm{F}$, \{sodass die Sache sicher in Ordnung geht $\} \mathrm{F}$.

Was die Fokus-Hintergrund-Gliederung von Satzverknüpfungen angeht, die durch Verbzweitsatz-Einbetter hergestellt werden, so ist sie nicht so frei wie die von Satzverknüpfungen, die durch Subjunktoren hergestellt werden, doch liegen die Dinge etwas komplizierter als bei den Postponierern. Aus Platzgründen kann ich hierauf nicht eingehen. Ich verweise diesbezüglich auf Pasch et al. (2003: Abschnitt C 1.3.4).

Indem Konjunktoren ihre Konnekte koordinieren, machen sie diese, wenn sie (wie in (18a)) Sätze sind, tauglich dafür, in ein und derselben syntaktischen Funktion verwendet zu werden (wie in (18b)):

Hat es Frost gegeben und die Dahlien sind schwarz geworden?

(18b) Weißt du, ob es Frost gegeben hat und die Dahlien schwarz geworden sind?

In (18b) üben die beiden (eingerahmten) Konnekte von und die syntaktische Funktion eines Komplements zum Verb wissen aus. In diesem Sinne ist zwischen seinen Konnekten stehendes denn, anders als traditionell unterstellt, kein koordinierender Konnektor: Anders als „echte“ koordinierende Konjunktionen wie und stellt denn, wie mit (16) gezeigt, keine Verknüpfung her, bei der beide satzförmigen Konnekte bei Einbettung in einen Satz dieselbe syntaktische Funktion ausüben könnten. Dies sieht man u.a. daran, dass bei denn anders als bei koordinierenden Konnektoren die Bedeutung von denn und die seines internen Konnekts nicht zusammen mit der seines externen Konnekts im Skopus eines Satzadverbials oder des Satzmodus des Satzes liegen kann, der die denn-Verknüpfung als Teilausdruck einschließt. Vgl. (6b) vs. (6b') vs. (18b): 
Wenn die Satzverknüpfung (6b') wohlgeformt werden soll, darf nur das erste Konnekt ein Fragesatz sein: Hat es Frost gegeben? Denn die Dahlien sind ganz schwarz.

Aufgrund dieses Befundes wird denn im Handbuch im Unterschied zu den koordinierenden Konnektoren (Konjunktoren) als ,parataktisch verknüpfender" Konnektor betrachtet, d.h. als ein Konnektor, der keine syntaktische Relation zwischen seinen Konnekten herstellt. Seine Konnekte sind als zwei syntaktisch selbständige Sätze zu betrachten.

\subsubsection{Syntaktische Klassen konnektintegrierbarer Konnektoren und ihre Merkmale}

$\mathrm{Zu}$ den konnektintegrierbaren Konnektoren gehören wie gesagt die relationalen Satzadverbien und die traditionell „Partikeln“ genannten Wortschatzeinheiten. Die meisten Grammatiken grenzen die Satzadverbien von den „Partikeln“ u.a. nach topologischen Kriterien ab. Beispiele hierfür sind Grundzüge (1981: 684ff.), Engel (1988: 861), GDS (1997: 54), Helbig/Buscha (1998: 341) und Eisenberg (1999: 209). Dabei wird darauf hingewiesen, dass „Adverbien“ das Vorfeld eines Verbzweitsatzes (in anderer Terminologie: eines „Aussagesatzes") besetzen können. In der Duden-Grammatik (1998: 87) finden sich in einem allgemeinen Abschnitt über Wortklassen zu „Adverbien“ überhaupt keine topologischen Angaben, sondern syntaktisch und semantisch funktionale (wie „als (notwendige) Ergänzung bzw. (freie) Angabe [...], als Prädikativum [...] oder als Attribut [...] gebraucht. Mit Adverbien werden - ganz allgemein gesprochen - in der Regel nähere Umstände [wovon? - R.P.] angegeben"). Topologische Angaben zu Adverbien finden sich in der Duden-Grammatik (1998) auch nicht im speziellen Adverbabschnitt auf S. $361 \mathrm{ff}$. Die Stellungsmöglichkeiten von Adverbien lassen sich in dieser Grammatik nur sehr indirekt über die Illustration möglicher Positionen von „Ergänzungen“ und „Angaben“" (s. ibid.: 628f.) ermitteln. (Bezüglich der Probleme, die die Bestimmung einer Wortklasse „Adverbien“ aufwirft, verweisen wir im Übrigen auf Eisenberg 1999: 205ff.)

Der Versuch, „Partikeln“ als Wortklasse zu bestimmen, wirft viele Probleme auf, auf die ich hier im Einzelnen aus Platzgründen nicht eingehen kann aber auch nicht eingehen muss, weil wir keine derartige syntaktische bzw. lexikalische Klasse ansetzen (zur Problematik s. Eisenberg 1999: 227ff.). Es sei nur so viel gesagt, dass in den Grammatiken unter diesem Terminus Einheiten unterschiedlicher Wortklassen zusammengeführt werden. Wir haben bis hierher dem Inhalt des von uns verwendeten Ausdrucks „Partikeln“ ein Merkmal zugrunde gelegt, das in einigen der oben genannten Grammatiken für lexikalische Einheiten als Kriterium dafür geltend gemacht wurde, dass sie zur Wortklasse „Partikeln“ gehören. Es ist dies das Merkmal, dass diese Einheiten nicht 
das Vorfeld besetzen können - vgl. Helbig/Buscha (1998: 475) und Grundzüge (1981: 497). Bei der letztgenannten Grammatik ist dieses Merkmal allerdings nur aus der Angabe abzuleiten, dass die betreffenden Einheiten „keinen Satzgliedwert" haben, wobei die „Satzgliedhaftigkeit sich als Möglichkeit definiert, u.a. das Vorfeld eines Verbzweitsatzes zu besetzen“ (s. Grundzüge 1988: 181). Die Duden-Grammatik (1998: 87) charakterisiert „Partikeln“ syntaktisch so: „Die Partikeln sind syntaktisch dadurch gekennzeichnet, dass sie keine Satzglieder bilden.“ Die Kenntnis ihrer Stellungseigenschaften muss sich der Benutzer der Grammatik durch eine hohe Eigenleistung erarbeiten: Er muss „Ergänzungen“ und „Angaben“ als „Satzglieder“ ausmachen und dann deren Stellungsmöglichkeiten aus den Beispielen für ihren Gebrauch ableiten und kann danach - hoffentlich - zufällig zu dem richtigen Ergebnis kommen, welches die möglichen und welches die nicht möglichen Positionen der „Partikeln“ sind. Die anderen oben genannten Grammatiken verwenden entweder überhaupt keinen Allgemeinbegriff „Partikel“ - wie die GDS (1997) und Eisenberg (1999) -, oder verwenden - wie Engel (1988: 877) - den Terminus für die Nichtflektierbaren ganz allgemein.

Mit der Unterscheidung der genannten zwei großen Klassen im Bereich der nichtflektierbaren satzintern verwendbaren Ausdrücke sind die Möglichkeiten, diese Ausdrücke nach syntaktischen Möglichkeiten zusammenzufassen, allerdings noch nicht erschöpft. Im Interesse einer Ökonomie der Beschreibung der lexikalischen Einheiten in Wörterbüchern sollte auf eine solche Subklassifizierung auch nicht verzichtet werden. Diesem Bedürfnis versuchen die Grammatiken offensichtlich auch gerecht zu werden. Allerdings werden die Subklassen in den genannten Grammatiken kaum nach syntaktischen, also wesentlich formalen kombinatorischen Kriterien bestimmt: Subklassen von Adverbien werden dort a) nach binnenstrukturellen Eigenschaften gebildet (wie „Pronominaladverb“, „abgeleitetes“, „diskontinuierliches“, „zusammengesetztes“ Adverb) und/oder b) nach semantischen (wie direktiv, final, instrumental, kausal, komitativ, konditional, lokal, modal, modifikativ und temporal; s. Grundzüge 1988: 692ff., Engel 1988: 861f., Duden-Grammatik 1998: 361., Helbig/Buscha 1998: 343ff, GDS 1997: 1174ff., die allerdings eine unterschiedliche Auswahl aus den genannten Klassenmerkmalen treffen). (In der Duden-Grammatik 1995: $358 \mathrm{ff}$. wechseln sogar unterschiedliche Klassifizierungskriterien bei Subklassen, die als alternative Klassen gleicher Ordnung präsentiert werden. So werden dort „Lokaladverbien“ wie hier, „Temporaladverbien“ wie jetzt, „Modaladverbien“ wie folgendermaßen, „Konjunktionaladverbien“ wie deshalb als relationale Adverbien, „Kommentaradverbien“ wie zweifellos und „Pronominaladverbien“ wie darauf, hierauf, worauf einander als Klassen gleicher Ordnung gegenübergestellt.) Subklassen von Partikeln werden traditionell ebenfalls angenommen, aber auch hier nach heterogenen Kriterien gebildet: nach semantischen Gesichtspunkten - vgl. „Modalpartikel“ -, nach prosodisch-positionell-informationsstrukturellen Kriterien - vgl. „Fokuspartikel“ - oder auch nach Bündeln von Eigenschaften unterschiedlichen Charakters, wie z.B. in der GDS (1997: 988) gemäß den Kriterien „nicht negierbar, nicht koordinierbar, 
nicht erfragbar, kein phrasales Kommutationsparadigma, nicht vorfeldfähig“ für die „Abtönungspartikeln“ genannten Ausdrücke.

Da zum einen als Konstituenten von Sätzen auftretende Konnektoren in unterschiedlichen Positionen im Satz vorkommen können, aber nicht alle derartigen Einheiten in sämtlichen für solche Einheiten ermittelbaren Positionen, zum anderen aber Kenntnisse der jeweils möglichen Positionen eines bestimmten Konnektors unabdingbar für seine korrekte Verwendung sind, erheben wir Stellungseigenschaften der betreffenden Konnektoren zum Kriterium für deren syntaktische Subklassifizierung. Dabei beschränken wir uns bei der Unterscheidung von „Adverbien“ und „Partikeln“ auf eine Unterscheidung nach Positionstypen, obwohl in den Grammatiken, die mit dieser Unterscheidung arbeiten, neben den positionellen weitere Unterscheidungskriterien angegeben werden. Eines davon ist - z.B. für Engel (1988: 861) - die Möglichkeit, dass zwar Adverbien, nicht aber andere satzintern zu verwendende nichtflektierbare einfache Ausdrücke als Antwort auf eine Ergänzungs- (w-) Frage fungieren. (Vgl. A.: Wann hast du das gemacht? B.: Gestern. (Adverb).) Es ist z.B. keine Frage vorstellbar, auf die Konnektoren, die nach dem Positionskriterium als Adverbien zu klassifizieren sind wie also, nichtsdestoweniger, nur als Antwort zählen könnten. Engel (1988: 879) nennt deshalb Ausdrücke, die zwar das Vorfeldkriterium, nicht aber das Erfragbarkeitskriterium erfüllen, ,Rangierpartikeln" und bestimmt Adverbien als Einheiten, die beide Kriterien erfüllen (s. ibid.: 861). Nun kann die Erfragbarkeit zwar zur Klassifizierung von Konnektoren herangezogen werden, da sie aber ein semantisches Kriterium ist, ziehen wir sie nicht zur Ermittlung syntaktischer Oberklassen heran. Wir wollen damit allerdings nicht ausschließen, dass es sinnvoll sein kann, im Rahmen der von uns vorgeschlagenen Oberklassen, die wir im Folgenden vorstellen werden, weitere Subklassen zu unterscheiden: nach weiteren positionellen Merkmalen weitere syntaktische Klassen, nach weiteren syntaktischen und/oder semantischen Kriterien weitere lexikalische Klassen.

Für die syntaktische Klassifizierung der nichtflektierbaren satzintern verwendbaren lexikalischen Einheiten, darunter auch der so charakterisierten Konnektoren, übernehmen wir die traditionelle Zweiteilung in „Adverbien“ und „Partikeln“ nicht als lexikographisch relevante Klassenbildung. Vielmehr unterscheiden wir drei rein topologisch fundierte syntaktische Klassen. In dieser Klassifikation werden die „Partikeln“ rein positionell als Einheiten definiert, die nicht das Vorfeld besetzen können, und die nach den oben angegebenen Positionsmöglichkeiten als „Adverbien“"klassifizierten Einheiten syntaktisch nach weiteren topologischen Eigenschaften in zwei Klassen gruppiert. Dadurch erscheint die Frage der Subklassifizierung von „Adverbien“ und „Partikeln“ in einem neuen Licht: Die Unterscheidung von Klassen auf der Grundlage nichtsyntaktischer Merkmale wird in der Syntax überflüssig.

Unser lexikographisch-praktischer Ansatz zur syntaktischen Subklassifizierung von Konnektoren, die satzintern verwendet werden können, ist folgender: Wir behandeln relationale Satzadverbien und Partikeln als Konnektoren, die in ihr internes Konnekt integriert verwendet werden können und deren 
externes Konnekt nicht in einer syntaktisch definierten Beziehung zum internen Konnekt steht. Dabei unterscheiden wir folgende syntaktische (= positionelle) Klassen konnektintegriert verwendbarer (,konnektintegrierbarer") Konnektoren:

„Nicht positionsbeschränkte Adverbkonnektoren“; diese müssen in folgenden Positionen verwendbar sein: Sie müssen a) das Vorfeld besetzen können, b) im Mittelfeld und c) in „Nacherstposition“ möglich sein; unter der "Nacherstposition“ verstehen wir die Position nach einer Konstituente im Vorfeld eines Verbzweitsatzes; vgl.:

(19a) [Das Buch ist jetzt erschienen,] allerdings hat es nur eine sehr kleine Auflage.

(19b) [Das Buch ist jetzt erschienen,] es hat allerdings nur eine sehr kleine Auflage.

(19c) [Das Buch ist noch nicht erschienen,] die Hypertextversion allerdings steht schon im Netz.

„Nicht nacherstfähige Adverbkonnektoren“; diese müssen in folgenden Positionen verwendbar sein: in a) Vorfeld- und b) Mittelfeldposition; sie dürfen c) nicht in Nacherstposition verwendet werden:

(20a) [Die Mannschaft verlor abermals.] Damit war ihr Schicksal besiegelt. (20b) [Die Mannschaft verlor abermals.] Ihr Schicksal war damit besiegelt.

(20c) [Die Mannschaft verlor abermals.] *Ihr Schicksal damit war besiegelt.

„Nicht vorfeldfähige Adverbkonnektoren“; diese müssen a) in Mittelfeldposition verwendbar sein und dürfen b) nicht das Vorfeld besetzen:

(21a) [Hans ist ausgesprochen vielseitig.] Er züchtet sogar Orchideen.

(21b) [Hans ist ausgesprochen vielseitig.] *Sogar züchtet er Orchideen.

Weitere mögliche, aber für uns nicht klassifizierungsrelevante Positionen der konnektintegrierbaren Konnektoren sind die „Vorerstposition“ (s. (22(a)), die Nachfeldposition (s. (22b)), die „Nullposition“ (s. (22c)) und die Nachsatzposition (s. (22d)).

(22a) [Ich kenne mehrere, die für die Aufgabe in Frage kommen.] Zum Beispiel Hans könnte man ja mal fragen.

(22b) [Ich kenne mehrere, die für die Aufgabe in Frage kommen.] Nehmen wir doch nur mal den Hans zum Beispiel.

(22c) [Viele Aufgaben blieben unerledigt.] Zum Beispiel: Niemand wollte die Partikeln analysieren.

(22d) [Viele Aufgaben blieben unerledigt.] Niemand wollte die Partikeln analysieren. Zum Beispiel. 
Diese Positionen sind bei den einzelnen konnektintegrierbaren Konnektoren in unterschiedlicher Verteilung und dabei auch nicht für alle konnektintegrierbaren Konnektoren gegeben und auch dann, wenn sie gegeben sind, nur bei einigen wenigen Konnektoren komplett.

\subsubsection{Allgemeingrammatische Leistungen des Handbuchs}

Neben dem Vorschlag für eine neue syntaktische Konnektorenklassifikation bringt das Handbuch als Beitrag zur Grammatikschreibung die Beschreibung von Phänomenen ein, die in den Gebrauchsbedingungen von Konnektoren und allgemeingrammatisch relevant sind, die aber bisher in den Grammatiken bei der Behandlung der Konnektoren zu wenig bis gar nicht berücksichtigt wurden. Zum Zwecke der möglichst umfassenden Beschreibung aller an den Gebrauchsbedingungen der Konnektoren beteiligten Aspekte sprachlicher Zeichen wurden im Handbuchprojekt Ergebnisse der derzeitigen Grammatikforschung - vor allem aus Spezialarbeiten - aus den unterschiedlichsten Bereichen von Syntax und Semantik zusammengetragen und integrativ verarbeitet. Dabei wurde, um den Nutzern den Inhalt der verwendeten Termini für die einschlägigen Phänomene zu vermitteln und ihnen die umständliche Konsultation von weiterer Literatur dazu zu ersparen, die Klärung von Begriffen im Handbuch selbst versucht. Weil wir, die Autoren des Handbuchs, die bisherige Behandlung einiger dieser Begriffe in der Literatur als unbefriedigend empfanden, erschien es uns erforderlich, uns unser eigenes Verständnis der betreffenden Begriffe zu erarbeiten und darzulegen - dies auch als Grundlegung für eine adäquate Beschreibung der semantischen und pragmatischen Eigenschaften der Konnektoren und damit für die Modellierung der Syntax-SemantikSchnittstelle im z.Z. laufenden Projektabschnitt mit dem geplanten Ergebnis eines Folgebandes zum Handbuch.

Bei den nicht nur für die Konnektorenbeschreibung relevanten von der Projektgruppe untersuchten und im Handbuch behandelten Phänomenen handelt es sich um folgende:

- $\quad$ Satz, Satzstruktur und Satzmodus

- Koordination, Subordination, Einbettung, Parataxe und Korrelatkonstruktionen

- $\quad$ Ellipsen

- die Gliederung (von Bedeutungen) von Sätzen in „Fokus“ vs. „Hintergrund“ im Zusammenhang mit prosodischen Eigenschaften sprachlicher Ausdrücke

- die Gliederung von Satzinterpretationen in eigentliche Satzbedeutung vs. für sie induzierte Präsuppositionen

- die Gliederung von Satzinterpretationen in propositionalen Gehalt vs. epistemischen Modus (auch „Einstellungsmodus“ genannt) und kommunikative Funktion (auch ,illokutive Kraft“ genannt) 
Ich kann hier nicht auf alle allgemeingrammatischen Erträge aus der Beschäftigung des Handbuchs mit den aufgelisteten Phänomenen im Detail eingehen. (Zur Behandlung der Gliederung in eigentliche Bedeutungen und Präsuppositionen im Handbuch s. detaillierter Max (in diesem Band).) Abschließend seien nur einige von ihnen herausgegriffen.

Ein allgemeinsyntaktischer Ertrag der Arbeit am Handbuch ist z.B. eine Liste von (größtenteils selbst ermittelten) Beschränkungen für die Weglassbarkeit von Konstituenten aus den im explizitesten Fall satzförmigen Konnekten von Konnektoren im Rahmen von Koordination und Subordination der Konnekte. Wir verweisen hierzu auf die Abschnitte B 6.3, B 5.7.5 und C 1.1.3.1.1 im Handbuch.

Als ein weiterer allgemeinsyntaktischer Ertrag ist vor allem die Unterscheidung zwischen "Subordination" in dem von uns intendierten Sinn und „Einbettung“ zu nennen. Die Unterscheidung ist nicht nur bei Konnekten nichtkonnektintegrierbarer Konnektoren notwendig, sondern auch bei Komplementsätzen - d.h. bei Sätzen, die als Ausdrücke für Aktanten von Verbbedeutungen fungieren, wo die subordinierten Sätze (d.h. Verbletztsätze) mehr Möglichkeiten bieten als die eingebetteten Verbzweitsätze. (Genaueres siehe Abschnitte B 5.1 bis B 5.3.) Vgl.:

(23a) [Morgen kommt Paul.] Ich weiß, er hat wenig Zeit.

(23b) [A.: Morgen kommt Paul. Er hat wenig Zeit. B.:] Dass er wenig Zeit hat, weiß ich. ${ }^{*}$ Er hat wenig Zeit, weiß ich.

Eine wichtige bislang in der Grammatiktradition kaum gemachte Unterscheidung (eine Ausnahme ist die Grammatik von Clément/Thümmel 1975), die wir Handbuch-Autoren im Zusammenhang mit der Thematik von Subordination und Einbettung für nötig erachten, ist dann die zwischen Sätzen (wie es interessiert sie, interessiert es sie und es sie interessiert) und Phrasen, die aus solchen Sätzen und sie regierenden Ausdrücken gebildet sind (wie vorausgesetzt, es interessiert sie; weil es sie interessiert; dass es sie interessiert; ob es sie interessiert). Diese Unterscheidung verlangt, dass Letztere nicht länger wie traditionell üblich (und wie auch noch in der GDS 1997: 2236 praktiziert) als „Nebensätze“ bezeichnet werden. Diese Bezeichnung versperrt das Verständnis dafür, warum zwar Verbzweitsätze wie es interessiert sie und Verberstsätze wie interessiert es sie, nicht aber Verbletztsätze wie es sie interessiert syntaktisch selbständig verwendet werden können, d.h. warum Letztere nur unmittelbar nach einem sie regierenden, d.h. subordinierenden Ausdruck (bzw. - bei Relativsätzen und indirekten Ergänzungsinterrogativsätzen - mit einem subordinierenden Ausdruck an ihrer Spitze) verwendet werden können und warum, wenn Konnektoren „Sätze“ regieren, nicht generell wiederum Ausdrücke aus Sätze subordinierenden Ausdrücken und dem von ihnen regierten Satz wie weil es sie interessiert, dass es sie interessiert, ob es sie interessiert als Kokonstituenten von Sätze regierenden Ausdrücken in Frage kommen (vgl. [sie kommt,] *ohne dass falls es sie interessiert, [das sage ich,] *falls dass es sie 
interessiert; [das sollte sie nur tun,] *wenn ob es sie interessiert (vgl. dagegen seitdem, dass es nicht mehr so kalt ist, wo seitdem als Pronominaladverb aufzufassen ist, das attributiv durch eine dass-Phrase erweitert ist).

Ein weiteres Phänomen, das wie schon in 6.1 gesagt bislang in den Grammatiken nicht gebührend berücksichtigt worden ist, ist die im Handbuch getroffene Unterscheidung zwischen der Koordination als Mittel der Bildung komplexer Sätze, die ihrerseits wieder Konstituenten noch komplexerer Sätze bilden können (vgl. die Beispiele unter (10)), von einem Phänomen, im Handbuch „Parataxe“ genannt, das als Verknüpfungstyp von Konnektoren wie nichtkonnektintegrierbarem denn („Begründungs-denn"; vgl. die abweichenden Konstruktionen unter (11)) angesetzt werden muss.

Ferner nimmt das Handbuch Stellung zu der in der Grammatikographie u.E. noch offenen Frage, welcher Art die syntaktische Beziehung zwischen den sog. Korrelaten und den eingebetteten Sätzen ist, auf die sich die Korrelate semantisch beziehen. Die Antwort auf diese Frage gründet sich auf die Unterscheidung zweier Typen von Korrelatkonstruktionen: attributiven Korrelatkonstruktionen und Versetzungskonstruktionen (s. hierzu im Detail HdK Abschnitt B 5.5).

Ein weiterer Punkt ist die Stellungnahme in einer Problematik, die in jüngster Zeit in der Grammatikforschung viel diskutiert wird, nämlich der mit semantischen Konsequenzen verbundenen Verwendung von Verberst- und Verbzweitsätzen nach Subjunktoren als Verbletztsätze regierenden Konnektoren. Vgl. gegenüber kanonischem (24a) nichtkanonisches (24b):

(24a) [Kontext: Es wird diskutiert, warum eine bestimmte Person für eine bestimmte Stelle ausgewählt wurde. Sprecher R.:] Das waren soziale Gründe. Weil ihr Stipendium abgelehnt wurde.

(24b) [Kontext: Es wird diskutiert, warum eine bestimmte Person für eine bestimmte Stelle ausgewählt wurde. Sprecher R.:] Det war'n soziale Gründe. Weil ihr Stipendium wurde abjelehnt. (Hörbeleg Oktober 2003)

Anders als in der Literatur üblich, wo die Meinung vorherrscht, dass bei den genannten Konstruktionsunterschieden unterschiedliche Konnektorenbedeutungen im Spiel sind, wird im Handbuch der Standpunkt vertreten, dass in solchen Fällen keine lexikalische Polysemie angenommen werden muss, sondern dass die semantischen Effekte, die sich aus der Verwendung von Verberst-/Verbzweitsätzen nach einem Subjunktor ergeben, in der Grammatik erklärt werden müssen, weil sie sich aus dem Zusammenspiel mehrerer Faktoren ergeben: aus einer einheitlichen Konnektorenbedeutung im Zusammenhang mit syntax- und prosodiebasierten semantischen Interpretationsregeln. Ein Argument für diese Sehweise ist, dass die betreffenden Konstruktionen nicht nur bei einem einzelnen Subjunktor vorkommen, sondern bei einer ganzen semantisch bestimmten Menge von Subjunktoren und Postponierern (kausalen, konzessiven und adversativen). (S. hierzu im Detail Abschnitt C 1.1.11.) 


\section{Anhänge}

\subsection{Zuordnung der nicht frei bildbaren Konnektoren zu den syntak- tischen Konnektorenklassen}

\section{Subjunktoren:}

abgesehen davon, dass; alldieweil; als; als ob; als wenn; angenommen, dass; anstatt; anstatt dass; anstelle dass; ausgenommen, dass; bevor; bis (dass); da; dadurch, dass; dafür, dass; damit; davon abgesehen, dass; dazu, dass; derweil(en); ehe; falls; gesetzt, dass; gesetzt den Fall, dass; gleichwohl; im Fall(e); indem; indes(sen); insofern; insofern (...), als; insoweit; insoweit (...), als; kaum dass; nachdem; nun; obgleich; obschon; obwohl; obzwar; ohne dass; seit(dem); sintemal(en); so; sobald; sofern; solang(e); sooft; sosehr; soviel; soweit; sowie; statt; statt dass; trotzderm; unterstellt, dass; vorausgesetzt, dass; während; währenddessen; weil; wenn; wenn (...) auch; wenngleich; wennschon; wie; wiewohl; wo; wofern; (zumal)

\section{Postponierer:}

als dass; ander(e)nfalls; auf dass; bloß dass; dass (final/konsekutiv); gdw.; nur dass; so dass/sodass; um so mehr, als; um so weniger, als; weshalb; weswegen; wobei; wodurch; wogegen; wohingegen; womit; wonach; worauf, woraufhin; zumal

\section{Verbzweitsatz-Einbetter:}

angenommen; für den Fall; gesetzt; gesetzt den Fall; im Fall(e); unterstellt; vorausgesetzt

\section{Konjunktoren:}

das heißt/d. h.ld.h.; d. i.; entweder (..) oder; ja; oder; respektivelresp.; sondern; sowie; sowohl (...) als (auch); sowohl (...) wie (auch); sprich; und; und/oder; will sagen

\section{Nicht positionsbeschränkte Adverbkonnektoren:}

allenfalls; allerdings; alsdann; also; and ((e)r)erseits; anders gesagt; ansonsten; beispielsweise; besonders; bestenfalls; blo $\beta$; bspw.; dafür; dagegen; dahingegen; dann; dementgegen; demgegenüber; derweil(en); einerseits; einesteils; endlich; freilich; genau gesagt; genauer gesagt; hingegen; hinwieder; hinwiederum; höchstens; immerhin; im Übrigen; indes(sen); insbesondere; in Sonderheit, jedenfalls; jedoch; kurz gesagt; m.a.W.; mindestens; mit anderen Worten; mithin; nichtsdestominder; nichtsdestotrotz; nichtsdestoweniger; 
noch1; nun; nur; obendrein; ohnehin; schließlich; schlussendlich; schon; sodann; überdies; überhaupt; übrigens; unterdes(sen); vor allem; währenddessen; wenigstens; wiederum; wohlgemerkt; z.B.; z.Bsp.; zudem; zu guter Letzt; zum Beispiel; zum einen; zumindest; zum Mindesten; zwar

\section{Nicht nacherstfähige Adverbkonnektoren:}

abermals; abgesehen davon; alldieweil; allemal; alsbald; ander(e)nfalls; ander(e)nteils; anfänglich; anfangs; anschließend; anstatt dessen; auch; außerdem; bald; bald (...), bald; da; dabei; dadurch; daher; damals; damit; danach; daneben; darauf, daraufhin; darüber hinaus; darum; davon abgesehen; davor; dazu; dazwischen; dementsprechend; demgemäß; demnach; demzufolge; dennoch; desgleichen; deshalb; dessen ungeachtet; desungeachtet; deswegen; des Weiteren; diesbezüglich; drauf, drum; ebenfalls; ebenso; einmal (...), ein andermal; entsprechend; ergo; erst $2(.)$, dann ; erstens (...), zweitens; erstmal; ferner; folglich; gegebenenfalls; genauso; gleichermaßen; gleichfalls; gleichwohl; gleichzeitig; halb (..), halb; hernach; hierbei; hierdurch; hiermit; hinterher; im Weiteren; insofern; insoweit; inzwischen; mal (...), mal; mittlerweile; nachher; nebenbei; nebenher; nunmehr; ohnedies; ohnehin; seitdem; seither; so; sofort; sogleich; so lang(e); somit; sonst; so weit; sowieso; später; stattdessen; teils (...), teils; trotzdem; um dessentwillen; vielmehr; von daher; vorher; weiter; weiterhin; weiters; zuerst; zugleich; zuletzt; zunächst; zusätzlich; zuvor; zwischendurch; zwischenzeitlich

\section{Nicht vorfeldfähige Adverbkonnektoren:}

aber; allein; ausschließlich; bereits; Fragen-denn; eh; einzig (und allein); erst ; etwa; gar; lediglich; nämlich; nicht (ein)mal; nur mehr; selbst; sogar; zumal

(Die Ziffern hinter manchen Konnektoren beziehen sich auf Varianten, die im Handbuch unterschieden werden. Ich verweise hierzu auf die Konnektorenliste in Pasch et al. (2003: Abschnitt D 2.), wo diese Varianten genauer beschrieben werden.)

Hierbei ist zu beachten, dass wir natürlich die schriftsprachlichen Varianten beispielsweise - bspw.; das heißt-d.h.ld. h.; mit anderen Worten - m.a.W.; zum Beispiel - z.B./z. B./z.Bsp/z. Bsp., die aus Gründen der alphabetischen Anordnung der Konnektoren in den Listen nicht in einem einzigen Eintrag aufgeführt sind, genauso wie die lautlichen und graphischen Varianten, die unter einem Vorkommen aufgeführt sind (vgl. z.B. ander $(e) n f a l l s ;$ and $((e) r) e r-$ seits; des(sen)ungeachtet, nurmehr/nur mehr; unterdes(sen)) nicht als jeweils eigenständige Konnektoren des deutschen Sprachsystems verstanden wissen wollen, sondern nur eben als Varianten ein und desselben Konnektors.

$\mathrm{Zu}$ den hier Klassen zugewiesenen Konnektoren gesellen sich noch die Konnektoren, die im Handbuch keiner Klasse zugeordnet werden, weil die 
Zuordnung zu einer Klasse keinen praktischen Nutzen bringen würde. Es handelt sich um folgende „Einzelgänger“ genannte Einheiten:

als mit folgendem konjunktivischem Verberstsatz; (an)statt; ausgenommen; außer; begründend-kausales dass; Begründungs-denn (d.h. denn, das nur zwischen seinen Konnekten zu verwenden ist); es sei denn; geschweige (denn); je nachdem; kaum (als temporaler Konnektor); $o b$ in syntaktisch desintegrierten Alternativenausdrücken; sei es; die konnektintegrierbaren Konnektoren beziehungsweiselbzw.; doch 1 und und zwar (mit den Merkmalen ,möglicher Vorfeldbesetzer' und ,nullstellenfähig'); doch2 (mit dem Merkmal, in Mittelfeldposition in einem Verberstsatz'); noch2 (mit Beschränkung auf die Vorfeldposition und einem Negationsausdruck im externen Konnekt, mehrheitlich weder) sowie wieder (mit den Merkmalen ,nur in Nacherstposition' und ,in adversativer Bedeutung") 
7.2 Matrix der syntaktischen Konnektorenklassen mit ihren Merkmalen

\begin{tabular}{|c|c|c|c|c|c|c|c|}
\hline $\begin{array}{l}\text { Klassenmerkmale nach } \\
\text { Dimensionen geordnet } \\
\end{array}$ & subj & post & v2eb & konj & npb & nne & nvf \\
\hline \multicolumn{8}{|l|}{$\begin{array}{l}\frac{1 . \text { bei Fehlen einer }}{\text { syntaktischen Relation }} \\
\text { zwischen den Konnekten: }\end{array}$} \\
\hline \multicolumn{8}{|l|}{$\begin{array}{l}\text { Position des Konnektors } K \\
\text { innerhalb des internen } \\
\text { Konnekts } k \#\end{array}$} \\
\hline a) $K$ besetzt das Vorfeld von $k \#$ & & & & & + & + & - \\
\hline b) $K$ im Mittelfeld von $k \#$ & & & & & + & + & + \\
\hline c) $K$ in Nacherstposition in $k \#:$ & & & & & + & - & 0 \\
\hline \multicolumn{8}{|l|}{$\begin{array}{l}\text { 2. bei Vorliegen einer } \\
\text { syntaktischen Relation } \\
\text { zwischen den Konnekten: }\end{array}$} \\
\hline \multicolumn{8}{|l|}{ 2.1. Position des Konnektors $K$ : } \\
\hline $\begin{array}{l}\text { a) } K \text { unmittelbar vor dem } \\
\text { internen Konnekt } k \#\end{array}$ & + & + & + & + & & & \\
\hline b) andere Positionen ungleich a) & - & - & - & - & & & \\
\hline \multicolumn{8}{|l|}{$\begin{array}{l}\text { 2.2. Syntaktisches Verhältnis } \\
\text { zwischen den Konnekten: }\end{array}$} \\
\hline a) $K$ bettet $k \#$ in $k$ a ein & + & - & + & - & & & \\
\hline b) $K$ subordiniert $k \# \operatorname{dem} k$ a & + & + & - & - & & & \\
\hline c) $K$ koordiniert $k \#$ und $k$ a & - & - & - & + & & & \\
\hline \multicolumn{8}{|l|}{ 2.3. Konnekteigenschaften: } \\
\hline a) $k \#$ Verbletztsatz & + & + & - & + & & & \\
\hline b) $k \#$ beliebiger Verbzweitsatz & 0 & 0 & - & + & & & \\
\hline c) $k \#$ konstativer Verbzweitsatz & 0 & 0 & + & + & & & \\
\hline d) $k \#$ Verberstsatz & 0 & 0 & - & + & & & \\
\hline e) $k \#$ nicht satzförmig & 0 & - & - & + & & & \\
\hline \multicolumn{8}{|l|}{ 2.4. Topologie der Konnekte: } \\
\hline $\begin{array}{l}\text { a) }\{K \text { und } k \#\} \text { besetzen das } \\
\text { Vorfeld von } k \text { a }\end{array}$ & + & - & + & - & & & \\
\hline $\begin{array}{l}\text { b) }\{K \text { und } k \#\} \text { im Mittelfeld von } \\
k \text { a }\end{array}$ & + & - & + & - & & & \\
\hline c) $\{K$ und $k \#\}$ nach $k$ a & + & + & + & + & & & \\
\hline d) $\{K$ und $k \#\}$ vor $k$ a & 0 & - & + & - & & & \\
\hline \multicolumn{8}{|l|}{ 2.5. Informationsstruktur: } \\
\hline Konnekte nur fokal & - & + & + & - & & & \\
\hline
\end{tabular}




\section{ERLÄUTERUNGEN:}

„subj“ für Subjunktoren (z.B. bevor)

„post" für Postponierer (z.B. sodass)

„v2eb“ für Verbzweitsatz-Einbetter (z.B. angenommen)

„konj“ für Konjunktoren (z.B. und)

„npb“ für nicht positionsbeschränkte Adverbkonnektoren (z.B.also)

„nne“ für nichtnacherstfähige Adverbkonnektoren (z.B. danach)

„nvf" für nicht vorfeldfähige Adverbkonnektoren (z.B.aber)

Aus Gründen der Vergleichbarkeit von nichtkonnektintegrierbaren mit konnektintegrierbaren Konnektoren wird in der Matrix eine zusätzliche, den anderen Dimensionierungen übergeordnete Dimension der Merkmalsortierung eingeführt, nämlich die von „Fehlen/Vorliegen einer syntaktischen Relation zwischen den Konnekten“.

Die folgenden Angaben in den Feldern unter den syntaktischen Konnektorenklassen sind wie folgt zu deuten:

„+“: Die Einheiten der betreffenden Klasse müssen das betreffende Merkmal erfüllen können.

„“: $\quad$ Das betreffende Merkmal ist für die Konnektoren der betreffenden Klasse ausgeschlossen.

„0“: In der betreffenden syntaktischen Klasse gibt es Elemente, die das betreffende Merkmal nicht aufweisen; deshalb wurde es nicht zum Klassenkriterium erhoben. Ob ein bestimmtes Element der Klasse das Merkmal aufweist oder nicht, ist dem Wörterbuch zu entnehmen.

Ein leeres Feld in der Matrix bedeutet, dass für die betreffende Konnektorenklasse die Voraussetzungen für die Zuschreibung des betreffenden Merkmals nicht erfüllt sind (weil höherrangige Merkmale nicht erfüllt sind).

Die Angabe „0“ bedarf, damit die Matrix voll interpretierbar wird, der Angabe, welche Elemente der betreffenden Klasse es sind, auf die das jeweilige Merkmal zutrifft. Ich führe im Folgenden bei den betreffenden Merkmalen in Frage kommende Konnektoren auf:

1. c): $\quad \boldsymbol{K}$ in Nacherstposition in $\boldsymbol{k} \#$ : nicht vorfeldfähige Adverbkonnektoren: aber, nämlich - vgl. z.B. Dort ist es im Sommer schön, im Winter aber kann es sehr kalt werden.

2.3. b): $k$ \# beliebiger Verbzweitsatz: Subjunktoren: weil; obwohl; während vgl. z.B. Da hab' ich gedacht um halb acht, die fangen da nämlich schon um halb acht an, während hier in der Stadt fängt es immer erst um acht an. (Hörbeleg 1997); Postponierer: wobei - vgl. Das is' Sandstein - wobei: Diese Sandsteinkugeln sind ab dieser Größe erst schön, aber dann kannste se nich' mehr bezahl'n. (Hörbeleg 1996); 2.3. b) impliziert 2.3. c). 
2.3. d): $k \#$ Verberstsatz: Subjunktoren: weil; obwohl; während - vgl. z.B. Ich kann dir soviel Geld nicht leihen, weil bin ich Krösus?/weil greif mal 'nem nackten Mann in die Tasche!; Postponierer: wobei - Das ist ja ganz schön, wobei: Gibt es nicht noch Besseres?

2.3. e): $\quad k \#$ nicht satzförmig: da; falls; obwohl; obschon; obzwar; weil; wenn; wenn (...) auch; wenngleich - vgl. Obwohl in diesen Dingen sehr beschlagen, kam er mit dem Problem nicht zurecht. oder Bist du fertig? Wenn nicht, gehe ich alleine.; Subjunktoren, die ein nicht satzförmiges $k \#$ nicht zulassen, sind z.B. die temporalen Subjunktoren (z.B. als; bevor; nachdem).

2.4. e): $\{\boldsymbol{K}$ und $\boldsymbol{k} \#\}$ vor $\boldsymbol{k}$ : Welche Subjunktoren diese Position erlauben und welche nicht, ist dem Wörterbuch zu entnehmen; möglich ist sie bei kausalen, konditionalen, konzessiven und temporalen Subjunktoren - vgl. z.B. Wenn es dir hier nicht gefällt, dann geh doch nach Hause,; nicht erlaubt ist sie z.B. bei ohne dass und (an-)statt dass.

Es sei hier noch darauf hingewiesen, dass die Subjunktoren, die das Merkmal unter 2.2. e) - $k \#$ nicht satzförmig - erfüllen können, dies nur mit spezifischen Einschränkungen tun. So kann sich zwar bei wenn und falls das interne Konnekt $k \#$ in seiner lautlichen Gestalt auf einen Satzmodifikator (wie z.B. nicht oder überhaupt) reduzieren, bei kausalen Subjunktoren ( $d a$ und weil) sowie konzessiven (obwohl; obschon; obzwar; wenn (...) auch; wenngleich) kann es sich dagegen nur als Nominal-, Partizipial- oder Adjektivphrase manifestieren.

\section{Literatur}

Blühdorn, Hardarik (2002a): Rauminformation und Demonstrativität. Am Beispiel des Deutschen. In: Deutsche Sprache 30, S. 252-275.

Blühdorn, Hardarik (2002b): Zur Negation im Deutschen und im Portugiesischen: nicht und kein, não und nenhum. In: Runa. Revista Portuguesa de Estudos Germanísticos 29, S. 267-296.

Blühdorn, Hardarik (2003a): Die Raumadverbien hier, da und dort und ihre Entsprechungen im brasilianischen Portugiesisch. In: Blühdorn, Hardarik/Schmidt-Radefeldt, Jürgen (Hgg.): Die kleineren Wortarten im Sprachvergleich Deutsch-Portugiesisch. Frankfurt a. M.: Peter Lang, S. 47-71.

Blühdorn, Hardarik (2003b): Zur Semantik der Konjunktion als. Paradigmatische und syntagmatische Aspekte. In: Linguistik online 13/1, S. 11-53. http://www.linguistik-online.com/13_01/bluehdorn.html

Blühdorn, Hardarik/Meireles, Selma M. (Ms.): Zur Semantik von Satzkonnektoren mit Negationskomponente.

Brauße, Ursula (1994a): Der Beitrag der Partikel auch zur Modifikation von Konditionalsätzen. In: Brauße, Ursula (Hg.): Lexikalische Funktionen der 
Synsemantika. Tübingen: Narr. (Forschungsberichte des Instituts für deutsche Sprache 71), S. 143-159.

Brauße, Ursula (1994b): Der Beitrag der Partikel denn in Konditionalsätzen. In: Brauße, Ursula (Hg.): Lexikalische Funktionen der Synsemantika. Tübingen: Narr. (Forschungsberichte des Instituts für deutsche Sprache 71), S. 159-168.

Brauße, Ursula (1994c): Funktionale Varianten von schon - Adverb, Gradpartikel, Modalpartikel. In: Brauße, Ursula (Hg.): Lexikalische Funktionen der Synsemantika. Tübingen: Narr. (Forschungsberichte des Instituts für deutsche Sprache 71), S. 99-118.

Brauße, Ursula (1994d): Lexikalische Funktionen der Synsemantika. Tübingen: Narr. (Forschungsberichte des Instituts für deutsche Sprache 171).

Brauße, Ursula (1997): Konnektoren im Wörterbuch. Konjunktionen, Adverbien, Partikeln. In: Konerding, Klaus-Peter/Lehr, Andrea (Hgg.): Linguistische Theorie und lexikographische Praxis. Symposiumsvorträge, Heidelberg 1996. Tübingen: Niemeyer. (Lexikographica Series Maior 82), S. 99108.

Brauße, Ursula (1998): Was ist Adversativität?: aber oder und? In: Deutsche Sprache 26/2, S. 138-159.

Brauße, Ursula (2000): Die Partikel allein. Klassifizierungs- und Bedeutungsprobleme. In: Linguistik online 6/2.

URL: http://www.linguistik-online.com/2_00/brausse.html

Brauße, Ursula (2001): Die kontextuellen Varianten des Konnektors doch. Ein Ausdruck von Relationen zwischen Widerspruch und Begründung. In: Kocsány, Piroska/Molnár, Anna (Hgg.): Wort und (Kon)text. Frankfurt a. M. u.a.: Peter Lang. (Metalinguistica 7), S. 151-171.

Brauße, Ursula (2002): Die Konjunktionen im de Gruyter Wörterbuch Deutsch als Fremdsprache. In: Wiegand, Herbert Ernst (Hg.): Perspektiven der pädagogischen Lexikographie des Deutschen II. Untersuchungen anhand des „de Gruyter Wörterbuchs Deutsch als Fremdsprache“. Tübingen: Niemeyer. (Lexicographica Series Maior 110), S. 339-348.

Breindl, Eva (1998): Konzeption und Konversion: zur simultanen Produktion von Printtext und Hypertext am Beispiel Grammatik. In: Storrer, Angelika/Harriehausen, Bettina (Hgg.): Hypermedia für Lexikon und Grammatik. Tübingen: Narr. (Studien zur deutschen Sprache 12), S. 75-98.

Breindl, Eva (2001): Rezension von: Pittner, Karin. Adverbiale im Deutschen. Untersuchungen zu ihrer Stellung und Interpretation. Tübingen: Stauffenburg Verlag 1999. In: Zeitschrift für Sprachwissenschaft 20/2, S. 280-286.

Breindl, Eva (2003a): Das Rätsel um das paradoxe allerdings: (K)ein Fall für die Textgrammatik der deutschen Sprache? In: Thurmair, Maria/Willkop, Eva (Hgg.): Am Anfang war der Text - 10 Jahre „Textgrammatik der deutschen Sprache“. München: iudicium, S. 73-94. 
Breindl, Eva (2003b): Alle sprechen von Lernergrammatik: und was ist mit den Lehrern? In: Wolff, Armin/Riedner, Ursula Renate (Hgg.): Grammatikvermittlung - Literaturreflexion - Wissenschaftspropädeutik - Qualifizierung für eine transnationale Kommunikation. Regensburg: Fachverband Deutsch als Fremdsprache. (Materialien Deutsch als Fremdsprache 70), S. 202-223.

Breindl, Eva (im Druck a): Konnektoren in Übungsgrammatiken. In: Kühn, Peter (Hg.): Übungsgrammatiken für Deutsch als Fremdsprache. Linguistische Analysen und didaktische Konzepte. Regensburg: Fachverband Deutsch als Fremdsprache. (Materialien Deutsch als Fremdsprache 66).

Breindl, Eva (im Druck b): Konzessivität und konzessive Konnektoren im Deutschen. Erscheint in: Deutsche Sprache.

Breindl, Eva (im Druck c): Polysemie und Invarianz bei Konnektoren: allerdings und andere Kontrastmarker. In: Pohl, Inge/Konerding, Klaus Peter (Hgg.): Prozesse der Bedeutungskonstruktion. Frankfurt a. M.: Lang.

Clément, Danièle/Thümmel, Wolf (1975): Grundzüge einer syntax der deutschen standardsprache. Frankfurt a. M. (Fischer Athenäum 2057).

Duden (1998): Grammatik der deutschen Gegenwartssprache. 6. Auflage. Mannheim: 6. Auflage Dudenverlag.

Fritsche, Johannes (Hg.) (1982): Konnektivausdrücke, Konnektiveinheiten. Grundelemente der semantischen Struktur von Texten. Hamburg: Buske. (Papiere zur Textlinguistik 30).

Grundzüge (1981): Grundzüge einer deutschen Grammatik. Von einem Autorenkollektiv unter der Leitung von Karl Erich Heidolph, Walter Flämig und Wolfgang Motsch. Berlin: Akademie-Verlag.

Kempcke, Günter/Pasch, Renate (1998): Die Konjunktionen in Langenscheidts Großwörterbuch Deutsch als Fremdsprache. In: Wiegand, Herbert Ernst (Hg.): Perspektiven der pädagogischen Lexikographie des Deutschen. Untersuchungen anhand von „Langenscheidts Großwörterbuch Deutsch als Fremdsprache“. Tübingen: Niemeyer. (Lexicographica Series Maior 86), S. 233-243.

Lang, Ewald (1982): Die Konjunktionen im einsprachigen Wörterbuch. In: Agricola, Erhard/Schildt, Joachim/Viehweger, Dieter (Hgg.): Wortschatzforschung heute. Aktuelle Probleme der Lexikologie und Lexikographie. Leipzig: Enzyklopädie, S. 72-106.

Métrich, René/Faucher, Eugène/Courdier, Gilbert (1993; 1995; 1998; 2002): Les Invariables Difficiles. Dictionnaire allemand-français des particules, connecteurs, interjections et „mots de la communication“, in vier Bänden. Nancy: Association des Nouveaux Cahiers d'Allemand.

Pasch, Renate (1994a): Benötigen Grammatiken und Wörterbücher des Deutschen eine Wortklasse „Konjunktionen“? In: Deutsche Sprache 22/2, S. 97 116. 
Pasch, Renate (1994b): Konzessivität von wenn-Konstruktionen. Tübingen: Narr. (Forschungsberichte des Instituts für deutsche Sprache 72).

Pasch, Renate (1995): Implikaturen im Bereich lexikalisch induzierter Präsuppositionen. In: Liedtke, Frank (Hg.): Implikaturen: Grammatische und pragmatische Analysen. Tübingen: Niemeyer. (Linguistische Arbeiten 343), S. 75-85.

Pasch, Renate (1997): Weil mit Hauptsatz - Kuckucksei im denn-Nest. In: Deutsche Sprache 25/3, S. 75-85.

Pasch, Renate (1999): Der subordinierende Konnektor wo: kausal und konzessiv? In: Freudenberg-Findeisen, Renate ( $\mathrm{Hg}$.): Ausdrucksgrammatik versus Inhaltsgrammatik. Linguistische und didaktische Aspekte der Grammatik. München: iudicium, S. 139-154.

Pasch, Renate (2000a): Vorschlag für die Neuordnung der „subordinierenden“ Konjunktionen des Deutschen. In: Lefèvre, Michel (Hg.): Subordination in Syntax, Semantik und Textlinguistik. Tübingen: Stauffenburg. (Eurogermanistik. Europäische Studien zur deutschen Sprache 15), S. 23-32.

Pasch, Renate (2000b): Zur Bedeutung von deutsch geschweige (denn). In: Linguistik online $6 / 2$, URL: http://www.linguistik-online.com/2_00/pasch.html

Pasch, Renate/Brauße, Ursula/Breindl, Eva/Waßner, Ulrich Hermann (2003): Handbuch der deutschen Konnektoren. Linguistische Grundlagen der Beschreibung und syntaktische Merkmale der deutschen Satzverknüpfer (Konjunktionen, Satzadverbien und Partikeln). Berlin, New York: de Gruyter. (Schriften des Instituts für Deutsche Sprache 9).

Waßner, Ulrich Hermann (2001a): „Halb zog sie ihn, halb sank er hin“ - Anmerkungen zu einem phraseologischen Konnektor des Deutschen. In: Waßner, Ulrich Hermann (Hg.): Lingua et Linguae. Festschrift für Clemens-Peter Herbermann. Aachen: Shaker, S. 447-469.

Waßner, Ulrich Hermann (2001b): Konnektoren und Anaphorika. In: Cambourian, Alain (Hg.): Textkonnektoren und andere textstrukturierende Einheiten. Tübingen: Stauffenburg. (Eurogermanistik 16), S. 33-46.

Waßner, Ulrich Hermann (2002): Geschlossene Klassen? In: Rapp, Reinhard (Hg.): Sprachwissenschaft auf dem Weg in das dritte Jahrtausend. Akten des 34. Linguistischen Kolloquiums in Germersheim 1999. Teil II: Sprache, Computer, Gesellschaft. Bern u.a.: Peter Lang, S. 635-643.

Weydt, Harald (Hg.) (1969): Abtönungspartikel. Die deutschen Modalwörter und ihre französischen Entsprechungen. Bad Homburg u.a.: Gehlen.

Weydt, Harald (Hg.) (1977): Aspekte der Modalpartikeln. Studien zur deutschen Abtönung. Tübingen: Niemeyer. (Konzepte der Sprach- und Literaturwissenschaft 23).

Weydt, Harald (Hg.) (1979): Die Partikeln der deutschen Sprache. Berlin, New York: de Gruyter. 
Weydt, Harald (Hg.) (1981): Partikeln und Deutschunterricht. Abtönungspartikeln für Lerner des Deutschen. Heidelberg: Groos.

Weydt, Harald (Hg.) (1989): Sprechen mit Partikeln. Berlin, New York: de Gruyter.

Weydt, Harald/Ehlers, Klaas-Hinrich (1987): Partikel-Bibliographie. Internationale Sprachenforschung zu Partikeln und Interjektionen. Frankfurt a. M. u.a.: Lang.

Weydt, Harald/Harden, Theo/Hentschel, Elke/Rösler, Dietmar (Hgg.) (1989): Kleine deutsche Partikellehre. Ein Lehr- und Übungsbuch für Deutsch als Fremdsprache. 3. Aufl. München: Klett.

Weydt, Harald/Hentschel, Elke (1983): Kleines Abtönungswörterbuch. In: Weydt, Harald (Hg.): Partikeln und Interaktion. Tübingen: Niemeyer. (Reihe Germanistische Linguistik 44), S. 3-24.

Zifonun, Gisela/Hoffmann, Ludger/Strecker, Bruno et al. (1997): Grammatik der deutschen Sprache. 3 Bände. Berlin, New York: de Gruyter. (Schriften des Instituts für deutsche Sprache 7). 